\title{
Hall and Ion Slip Effects on Mixed Convection Flow of Eyring-Powell Nanofluid over a Stretching Surface
}

\author{
Wubshet Ibrahim $\mathbb{D D}^{1}$ and Temesgen Anbessa ${ }^{2}$ \\ ${ }^{1}$ Department of Mathematics, Ambo University, Ambo, Ethiopia \\ ${ }^{2}$ Department of Mathematics, Wollega University, Nekemte, Ethiopia \\ Correspondence should be addressed to Wubshet Ibrahim; wubshetib@yahoo.com
}

Received 27 June 2020; Revised 18 August 2020; Accepted 29 August 2020; Published 9 September 2020

Academic Editor: Shuo Yin

Copyright (c) 2020 Wubshet Ibrahim and Temesgen Anbessa. This is an open access article distributed under the Creative Commons Attribution License, which permits unrestricted use, distribution, and reproduction in any medium, provided the original work is properly cited.

\begin{abstract}
The purpose of this research is to inspect the mixed convection flow of Eyring-Powell nanofluid over a linearly stretching sheet through a porous medium with Cattaneo-Christov heat and mass flux model in the presence of Hall and ion slip, permeability, and Joule heating effects. Proper similarity transforms yield coupled nonlinear differential systems, which are solved using the spectral relaxation method (SRM). The story audits show that the present research problem has not been studied until this point. Efficiency of numerous parameters on velocity, temperature, and concentration curves is exposed graphically. Likewise, the numerical values of skin friction coefficients, local Nusselt, and Sherwood numbers are computed and tabulated for some physical parameters. It is manifested that fluid velocities, skin friction coefficients, local Nusselt, and Sherwood numbers promote with the larger values of Eyring-Powell fluid parameter $\varepsilon$. It is also noticed that primary velocity promotes with larger values of mixed convection parameter $\lambda$, Hall parameter $\beta_{e}$, and ion slip parameter $\beta_{i}$, while the opposite condition is observed for secondary velocity, temperature, and concentration. Furthermore, comparative surveys between the previously distributed writing and the current information are made for explicit cases, which are examined to be in a marvelous understanding.
\end{abstract}

\section{Introduction}

Mixed convection streams emerge in numerous transport processes both in nature and in engineering applications. They play a vital role, for instance, in air limit layer streams, heat exchangers, atomic reactors, solar collectors, and in electronic hardware. Such procedures happen when the impacts of buoyancy forces in constrained convection or the impacts of the constrained stream in natural convection become noteworthy. The interaction of natural and constrained convection is particularly articulated in circumstances where the constrained stream velocity is low as well as the temperature contrasts are huge. This stream is likewise an important kind of stream showing up in numerous mechanical procedures, for example, assembling and extraction of polymer and elastic sheets, paper creation, wire drawing and glass-fiber creation, dissolve turning, and consistent throwing. Moreover, mixed convection in permeable media has numerous applications, for example, food handling and storage, metallurgy, geophysical framework, fibrous insulation, and underground removal of atomic waste. Then again, thermal conductivity of the ordinary heat transport liquids, for instance, water, oil, and ethylene glycol are exceptionally low. Thus, expanding thermal conductivity of the traditional liquids prompts improves the heat transport of these liquids. As of late, nanofluids are presented with upgraded thermal conductivity. A nanofluid is a suspension of nanoparticles with normal sizes beneath $100 \mathrm{~nm}$ in the base liquids. Due to the upgraded thermal conductivity, nanofluids are proposed for some mechanical applications, for example, transportation, atomic reactors, and nourishment. In this manner, numerous ongoing analysts have generous enthusiasm for the mixed convection stream of nanofluid over an extending sheet in a permeable medium due to their impressive use in the modern and innovative applications. Accordingly, Ameen et al. [1] investigated a 3D turning stream of carbon nanotubes (CNTs) over a permeable stretchable sheet for warmth and mass exchange with thought of kerosene oil as a base fluid 
and announced that both velocity fields delineate expanding conduct for enormous value of the CNT nanoparticle. Besides, Rasool et al. [2] examined a numerical investigation of the MHD Williamson nanofluid flow maintained to flow through permeable medium bounded by a nonlinearly stretching flat surface and reported that an enhancement in the values of magnetic parameter augments the drag force, whereas a decrement in the drag force can be observed as the values of Weissenberg number enhances. Also, Ibrahim and Anbessa [3] examined the mixed convection stream of a Maxwell nanofluid over a growing surface in a penetrable medium utilizing the spectral relaxation method (SRM) and found that a climb in Deborah number decreases both the stream and transverse velocity profiles, while the backwards design is seen with enlargement in the mixed convection parameter. Furthermore, Waini et al. [4] examined the consistent mixed convection stream along a vertical surface implanted in a permeable medium with hybrid nanoparticles utilizing the bvp4c solver in Matlab programming. They considered both assisting and opposing streams and found that there exist dual solutions for the case of opposing stream. Further, numerous other ongoing works have been done around there as found in references [4-12].

Investigation of nonNewtonian liquid has incredible significance because of its numerous industrial and engineering applications. Specifically, these liquids are used in the material preparing, concoction and atomic ventures, bioengineering, oil repository designing, polymeric fluids, and groceries. A few liquids like paints, paper mash, shampoos, ketchup, fruit purée, slurries, certain oils, and polymer arrangements are instances of nonNewtonian liquids. NonNewtonian liquids are logically perplexed when compared with Newtonian liquids due to nonlinear association among the stress and strain rate. Various models have been proposed in the writing for the examination of nonNewtonian fluids; however, not a sole model is built up that shows all properties of nonNewtonian liquids. In general, nonNewtonian fluids are mainly characterized into three kinds: to be explicit differential, rate, and integral kinds. Among the nonNewtonian liquid models, the Eyring-Powell model has accomplished exceptional consideration of the analysts because of its distinctive attributes in current science. This could be derived from kinetic theory of liquids rather than from its empirical relation. Likewise, the Eyring-Powell model decreases to Newtonian liquid qualities for low and high shear rate. Warmth and mass exchange in the Eyring-Powell liquid model assumes a significant role in the procedures which include formation and spread of haze, plotting of concoction handling instrumentation, environmental contamination, drying of permeable slides, raised oil recuperation, warm protection, and underground energy transport. Khan et al. [13] explored blended convection stream of the Eyring-Powell liquid model with variable viscosity and convective limit conditions over a slanted extending sheet utilizing the homotopy analysis method (HAM) and revealed that velocity profile increments by expanding liquid parameter $M$ and Grashof numbers $G m$ and $G r$, while velocity profile diminishes by expanding variable viscosity parameter $A$, liquid parameter $k$, and magnetic field parameter $\mathrm{Ha}$. Moreover, Khan et al. [14] examined warm disper- sion and dissemination thermo impacts on the wobbly flow of electrically conducting Eyring-Powell liquid over an oscillatory extending sheet with convective limit conditions utilizing HAM and found that the bigger values of Eyring-Powell liquid parameter improve the amplitude of velocity and limit the layer thickness. However, inverse impacts are seen in temperature and concentration profiles. Furthermore, Ishaq et al. [15] explored two dimensional nanofluid film stream of Eyring-Powell liquid with variable warmth transmission in the presence of MHD on a shaky permeable extending sheet and announced that porosity parameter diminishes the movement of the fluid movies, and enlarging the nanoparticle concentration effectively expands the rubbing characteristic of Eyring-Powell nanofluid. In recent times, various researches have been made in the field of EyringPowell nanofluids that can be found in references [16-23].

Heat transport in the presence of strong magnetic field is significant in different parts of MHD power generation, nanotechnological processing, nuclear energy systems exploiting fluid metals, and blood stream control. Further, Hall and ion slip impacts become noteworthy in strong magnetic fields and can impressively influence the current density in hydromagnetic heat transport. Most recently, Krishna and Chamkha [24] explored Hall and ion slip impacts on the MHD free convective pivoting stream of nanofluids in a permeable medium past a moving vertical semiboundless level plate and announced the velocity increments with Hall and ion slip parameters. Furthermore, Rani et al. [25] explored Hall and ion slip impacts on the MHD natural convective pivoting stream of Ag-water-based nanofluid past a semiboundless penetrable moving plate with consistent warmth source utilizing the perturbation method. They found that in the limit layer area, liquid velocity diminishes with the expanding values of rotation parameter, magnetic field parameter, and suction parameter, while it increments with the expanding values of Hall and ion slip parameters. Further examinations identified with the MHD stream issues with Hall and ion slip impacts can be found in references [26-31].

The temperature differences between two distinct bodies cause heat transport device. It assumes a significant task in the creation of energy, cooling of atomic reactors, and biomedical applications such as medication targeting and heat conduction in tissues. Fourier [32] was the spearheading individual who at the outset portrayed the marvels of heat transport, which is the parabolic energy equation for temperature field and has disadvantage that initial interruption is felt right away all through the entire medium. Later on, so as to control this limitation, Cattaneo [33] improved the Fourier law of heat conduction by including the thermal relaxation term which causes heat transportation as thermal waves with limited speed. Furthermore, Christov [34] utilized Oldroyd's upper convected derivative instead of time derivative so as to achieve the material-invariant detailing. This new model is named as the Cattaneo-Christov heat flux model. After the spearheading work of Christov [34], a number of ongoing investigations in this setting are given in the references [35-45].

The aforesaid writing surveys affirmed that no endeavor has been made at this point to investigate the MHD mixed 
convection flow of Eyring-Powell nanofluid past a vertical linearly extending sheet through a porous medium in the presence of the Cattaneo-Christov heat and mass flux model with the consolidated effects of Hall and ion slip, porosity, and Joule heating. With this perspective, the current correspondence aims to fill this gap inside the current writing. Consequently, the fundamental object of the present work is to examine the impact of Hall and ion slip on the mixed convection stream of Eyring-Powell nanofluid past a vertical linearly extending sheet through a penetrable medium in the presence of Joule heating with the Cattaneo-Christov heat and mass transition model. Numerical clarifications were accomplished employing the spectral relaxation method [3, 46-48]. The effects of embedding parameters on the velocity, temperature, and concentration fields are analyzed graphically. Moreover, numerical estimations of skin friction coefficients, local Nusselt, and Sherwood numbers for some pertinent parameters are computed and tabulated.

\section{Mathematical Formulations}

Considering a laminar, steady, viscous, and incompressible mixed convection stream of an electrically conducting Eyring-Powell nanofluid past a vertical linearly extending sheet through a porous medium with velocity $U_{w}(x)=a x$ (where $a$ is a positive constant) toward $x$-axis, the CattaneoChirstov heat and mass flux model are utilized to explore the heat and mass transfer qualities. The surface temperature $T_{w}$ and concentration $C_{w}$ are assumed consistent at the extending surface. As $y$ extends to infinity, the encompassing values of temperature and concentration are, respectively, $T_{\infty}$ and $C_{\infty}$. Besides, a strong uniform magnetic field $B_{0}$ is applied in the direction along $y$-axis as shown in Figure 1. Because of this strong magnetic field force, the electrically conducting liquid has the Hall and ion slip impacts which produces a crossflow in the $z$-direction, and consequently, the stream becomes three dimensional. The $x$-axis is along the course of movement of the surface, $y$-axis is perpendicular to the surface, and $z$-axis is normal to the $x y$ plane. The induced magnetic field can be overlooked conversely with the applied magnetic field on the assumption that the stream is steady, and the magnetic Reynolds number is very little.

The extra stress tensor $\tau_{i j}$ of the Eyring-Powell liquid model is characterized as $[15,21]$ :

$$
\tau_{i j}=\mu \frac{\partial u_{i}}{\partial x_{j}}+\frac{1}{\beta} \sin ^{-1}\left(\frac{1}{b} \frac{\partial u_{i}}{\partial x_{j}}\right)
$$

where $\mu$ is the dynamic viscosity, and $\beta$ and $b$ are the material fluid parameters of the Eyring-Powell fluid model such that

$$
\sin ^{-1}\left(\frac{1}{b} \frac{\partial u_{i}}{\partial x_{j}}\right) \cong \frac{1}{b} \frac{\partial u_{i}}{\partial x_{j}}-\frac{1}{6}\left(\frac{1}{b} \frac{\partial u_{i}}{\partial x_{j}}\right)^{3} \text { and }\left|\frac{1}{b} \frac{\partial u_{i}}{\partial x_{j}}\right| \ll 1
$$

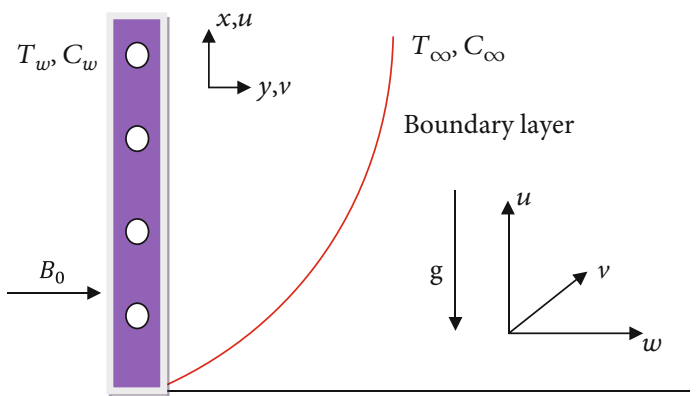

Figure 1: Physical representation of the flow problem.

Generalized Ohm's law with Hall and ion slip impacts is given by $[20,23]$

$$
\mathbf{J}=\sigma(\mathbf{E}+(\mathbf{V} \times \mathbf{B}))-\frac{\omega_{e} \tau_{e}}{B_{0}}(\mathbf{J} \times \mathbf{B})+\frac{\omega_{e} \tau_{e} \beta_{i}}{B_{0}^{2}}((\mathbf{J} \times \mathbf{B}) \times \mathbf{B}),
$$

where $\mathbf{J}=\left(J_{x}, J_{y}, J_{z}\right)$ is the current density vector, $\mathbf{E}$ is the intensity vector of the electric field, $\mathbf{V}$ is the velocity vector, $\mathbf{B}$ is the magnetic field, $\omega_{e}$ is the cyclotron frequency, and $\tau_{e}$ is the electrical collision time.

Applying the Boussinesq approximations alongside the above suppositions, the basic governing equations are $[30,36,43,44,49]$

$$
\begin{aligned}
\frac{\partial u}{\partial x}+\frac{\partial v}{\partial y}=0 & \\
u \frac{\partial u}{\partial x}+v \frac{\partial u}{\partial y}= & {\left[\left(v+\frac{1}{\beta b \rho}\right)-\frac{1}{2 \beta b^{3} \rho}\left(\frac{\partial u}{\partial y}\right)^{2}\right] \frac{\partial^{2} u}{\partial y^{2}} } \\
& -\frac{\sigma B_{0}^{2}}{\rho\left(\alpha_{e}^{2}+\beta_{e}^{2}\right)}\left(\alpha_{e} u+\beta_{e} w\right)+\mathrm{g}\left[\beta_{T}\left(T-T_{\infty}\right)\right. \\
& \left.+\beta_{C}\left(C-C_{\infty}\right)\right]-\frac{v}{k} u \\
u \frac{\partial w}{\partial x}+v \frac{\partial w}{\partial y}= & {\left[\left(v+\frac{1}{\beta b \rho}\right)-\frac{1}{2 \beta b^{3} \rho}\left(\frac{\partial w}{\partial y}\right)^{2}\right] \frac{\partial^{2} w}{\partial y^{2}} } \\
& +\frac{\sigma B_{0}^{2}}{\rho\left(\alpha_{e}^{2}+\beta_{e}^{2}\right)}\left(\beta_{e} u-\alpha_{e} w\right)-\frac{v}{k} w \\
u \frac{\partial T}{\partial x}+v \frac{\partial T}{\partial y}=\alpha & \frac{\partial^{2} T}{\partial y^{2}}-\gamma_{T}\left[\left(u \frac{\partial u}{\partial x}+v \frac{\partial u}{\partial y}\right) \frac{\partial T}{\partial x}+\left(u \frac{\partial v}{\partial x}+v \frac{\partial v}{\partial y}\right) \frac{\partial T}{\partial y}\right. \\
& \left.+2 u v \frac{\partial^{2} T}{\partial x \partial y}+u^{2} \frac{\partial^{2} T}{\partial x^{2}}+v^{2} \frac{\partial^{2} T}{\partial y^{2}}\right] \\
& +\tau\left[D_{B}\left(\frac{\partial T}{\partial y}\right)\left(\frac{\partial C}{\partial y}\right)+\left(\frac{D_{T}}{T_{\infty}}\right)\left(\frac{\partial T}{\partial y}\right)^{2}\right] \\
& +\frac{\sigma B_{0}^{2}}{\rho C_{p}\left(\alpha_{e}^{2}+\beta_{e}^{2}\right)}\left(u^{2}+w^{2}\right)
\end{aligned}
$$




$$
\begin{aligned}
u \frac{\partial C}{\partial x}+v \frac{\partial C}{\partial y}= & D_{B} \frac{\partial^{2} C}{\partial y^{2}}+\left(\frac{D_{T}}{T_{\infty}}\right)\left(\frac{\partial^{2} T}{\partial y^{2}}\right) \\
& -\gamma_{C}\left[\left(u \frac{\partial u}{\partial x}+v \frac{\partial u}{\partial y}\right) \frac{\partial C}{\partial x}+\left(u \frac{\partial v}{\partial x}+v \frac{\partial v}{\partial y}\right) \frac{\partial C}{\partial y}\right. \\
& \left.+2 u v \frac{\partial^{2} C}{\partial x \partial y}+u^{2} \frac{\partial^{2} C}{\partial x^{2}}+v^{2} \frac{\partial^{2} C}{\partial y^{2}}\right]
\end{aligned}
$$

with the relating boundary conditions $[3,36]$ :

$$
\begin{gathered}
u=U_{w}(x)=a x, v=w=0, T=T_{w}, C=C_{w} \text {, at } y=0, \\
u \rightarrow 0, \quad w \rightarrow 0, \quad T \rightarrow T_{\infty}, \quad C \rightarrow C_{\infty}, \quad \text { as } \quad y \rightarrow \infty,
\end{gathered}
$$

$$
\eta=\sqrt{\frac{a}{v}} y, \quad \psi=\sqrt{\operatorname{av} x} f(\eta), \quad w=\operatorname{axg}(\eta), \quad \theta(\eta)=\frac{T-T_{\infty}}{T_{w}-T_{\infty}}, \quad \phi(\eta)=\frac{\mathrm{C}-\mathrm{C}_{\infty}}{\mathrm{C}_{w}-\mathrm{C}_{\infty}}
$$

where $\eta$ is the similarity variable, and $\psi$ is the stream function defined as

$$
u=\frac{\partial \psi}{\partial y} \text { and } v=-\frac{\partial \psi}{\partial x}
$$

Equation (4) is identically fulfilled, and equations (5)-(9) become

$$
\begin{aligned}
& (1+\varepsilon) f^{\prime \prime \prime}-(\varepsilon \delta) f^{\prime \prime 2} f^{\prime \prime \prime}-f^{\prime 2}+f f^{\prime \prime}-\frac{M}{\left(\alpha_{e}^{2}+\beta_{e}^{2}\right)}\left(\alpha_{e} f^{\prime}+\beta_{e} g\right) \\
& +\lambda(\theta+N r \phi)-K f^{\prime}=0
\end{aligned}
$$

$$
\begin{aligned}
& (1+\varepsilon) g^{\prime \prime}-(\varepsilon \delta) g^{\prime 2} g^{\prime \prime}-f^{\prime} g+f g^{\prime} \\
& +\frac{M}{\left(\alpha_{e}^{2}+\beta_{e}^{2}\right)}\left(\beta_{e} f^{\prime}-\alpha_{e} g\right)-K g=0
\end{aligned}
$$

$$
\begin{aligned}
& \frac{1}{\operatorname{Pr}} \theta^{\prime \prime}+f \theta^{\prime}-\gamma_{1}\left(f f^{\prime} \theta^{\prime}+f^{2} \theta^{\prime \prime}\right)+N b \theta^{\prime} \phi^{\prime}+N t \theta^{\prime 2} \\
& \quad+\frac{M \cdot E c}{\left(\alpha_{e}^{2}+\beta_{e}^{2}\right)}\left(f^{\prime 2}+g^{2}\right)=0, \\
& \phi^{\prime \prime}+\operatorname{Pr} \cdot \operatorname{Le}\left[f \phi^{\prime}-\gamma_{2}\left(f f^{\prime} \phi^{\prime}+f^{2} \phi^{\prime \prime}\right)\right]+\frac{N t}{N b} \theta^{\prime \prime}=0 .
\end{aligned}
$$

where $u, v$, and $w$ are the velocity components along the $x$ ,$- y-$, and $z$-directions, respectively, and $v$ is the kinematic viscosity, $\rho$ is the density of the fluid, $\mathrm{g}$ is the acceleration due to gravity, $\beta_{e}$ is the Hall parameter, $\beta_{i}$ is the ion-slip parameter, $\alpha_{e}=1+\beta_{e} \beta_{i}$ is a constant, $B_{T}$ is the coefficient of thermal expansion, $B_{C}$ is the solutal coefficient of expansion, $\kappa$ is the permeability of the porous medium, $\mathrm{T}$ and $\mathrm{C}$ are the fluid temperature and concentration, respectively, $\gamma_{T}$ and $\gamma_{C}$ are the heat and mass flux relaxation times correspondingly, $C_{p}$ is the specific heat capacity, $\tau$ is the ratio of the effective heat capacity of nanoparticle to the heat capacity of the fluid, i.e., $\tau=(\rho \mathrm{C})_{p} /(\rho \mathrm{C})_{f}, D_{B}$ is the Brownian diffusion coefficient, and $D_{T}$ is the thermophoresis diffusion coefficient.

The overseeing equations can be changed to coupled nonlinear ordinary differential equations utilizing the subsequent similarity transformations $[3,36]$ :

The transformed boundary conditions are

$$
\begin{aligned}
& f(0)=0, f^{\prime}(0)=1, g(0)=0, \theta(0)=1, \phi(0)=1, \\
& f^{\prime} \longrightarrow 0, \quad g \longrightarrow 0, \quad \theta \longrightarrow 0, \quad \phi \longrightarrow 0, \quad \text { as } \eta \longrightarrow \infty .
\end{aligned}
$$

The dimensionless parameters showing up in equations (12)-(15) can be characterized as

$M=\sigma B_{0}^{2} / \rho a, \alpha_{e}=1+\beta_{e} \beta_{i} \lambda=G r_{x} / \operatorname{Re}_{x}^{2}, G r_{x}=g \beta_{T}\left(T_{w}-\right.$ $\left.T_{\infty}\right) x^{3} / v^{2}, \quad \operatorname{Re}_{x}=a x^{2} / v, \quad N r=\beta_{C}\left(\mathrm{C}_{w}-\mathrm{C}_{\infty}\right) / \beta_{T}\left(T_{w}-T_{\infty}\right)$, $K=v / a \kappa, \varepsilon=1 / \beta b \mu, \delta=a^{3} x^{2} / 2 b^{2} v \operatorname{Pr}=v / \alpha, N b=\tau D_{B}\left(C_{w}-\right.$ $\left.\mathrm{C}_{\infty}\right) / v, \quad N t=\tau D_{T}\left(T_{w}-T_{\infty}\right) / v T_{\infty}, \quad \tau=(\rho \mathrm{C})_{p} /(\rho \mathrm{C})_{f}, \quad E c=$ $(a x)^{2} / C_{p}\left(T_{w}-T_{\infty}\right), L e=\alpha / D_{B}, \gamma_{1}=\gamma_{T} a$ and $\gamma_{2}=\gamma_{C} a$.

The skin friction coefficients $C_{f x}$ and $C_{g z}$, the local Nusselt number $N u_{x}$, and the Sherwood number $S h_{x}$ are the physical quantities of interest which can be characterized as follows:

$C_{f x}=\frac{\tau_{w x}}{\rho U_{w}^{2}}, \quad C_{f z}=\frac{\tau_{w z}}{\rho U_{w}^{2}}, \quad N u_{x}=\frac{x q_{w}}{k\left(T_{W}-T_{\infty}\right)}, \quad S h_{x}=\frac{x j_{m}}{D_{B}\left(C_{W}-C_{\infty}\right)}$,

where $\tau_{w x}$ and $\tau_{w z}$ are the wall shear stresses in the directions of $x$ and $z$, respectively, and $q_{w}$ is the surface 
TABle 1: Comparison of results for the Nusselt number $-\theta^{\prime}(0)$ and Sherwood number $-\phi^{\prime}(0)$ for various values of $N b$ and $N t$ when $L e=1$, $\operatorname{Pr}=10$, and $M=\lambda=\beta_{e}=\beta_{i}=N r=\varepsilon=\delta=\gamma_{1}=\gamma_{2}=K=E c=0$ are fixed.

\begin{tabular}{|c|c|c|c|c|c|c|c|c|c|}
\hline \multirow[t]{2}{*}{$\mathrm{Nb}$} & \multirow{2}{*}{$N t$} & \multicolumn{2}{|c|}{$\begin{array}{c}\text { Noghrehabadi et al. } \\
\text { [51] }\end{array}$} & \multicolumn{2}{|c|}{$\begin{array}{l}\text { Goyal and Bhargava } \\
{[52]}\end{array}$} & \multicolumn{2}{|c|}{$\begin{array}{c}\text { Ibrahim and Gadisa } \\
{[53]}\end{array}$} & \multicolumn{2}{|c|}{ Present results } \\
\hline & & $-\theta^{\prime}(0)$ & $-\phi^{\prime}(0)$ & $-\theta^{\prime}(0)$ & $-\phi^{\prime}(0)$ & $-\theta^{\prime}(0)$ & $-\phi^{\prime}(0)$ & $-\theta^{\prime}(0)$ & $-\phi^{\prime}(0)$ \\
\hline \multirow[t]{5}{*}{0.1} & 0.1 & 0.9523768 & 2.1293938 & 0.95244 & 2.12949 & 0.95239 & 2.12938 & 0.9523768 & 2.1293938 \\
\hline & 0.2 & 0.6931743 & 2.2740215 & 0.69318 & 2.27401 & 0.69318 & 2.27401 & 0.6931743 & 2.2740216 \\
\hline & 0.3 & 0.5200790 & 2.5286382 & 0.52025 & 2.52855 & 0.52019 & 2.52855 & 0.5200790 & 2.5286387 \\
\hline & 0.4 & 0.4025808 & 2.7951701 & 0.40260 & 2.79520 & 0.40258 & 2.79519 & 0.4025823 & 2.7951628 \\
\hline & 0.5 & 0.3210543 & 3.0351425 & 0.32105 & 3.03511 & 0.32109 & 3.03511 & 0.3210491 & 3.0351686 \\
\hline 0.2 & 0.1 & 0.5055814 & 2.3818706 & 0.50561 & 2.38186 & 0.50557 & 2.38190 & 0.5055815 & 2.3818706 \\
\hline 0.3 & & 0.2521560 & 2.4100188 & 0.25218 & 2.41009 & 0.25217 & 2.41002 & 0.2521562 & 2.4100188 \\
\hline 0.4 & & 0.1194059 & 2.3996502 & 0.11940 & 2.39970 & 0.11940 & 2.39966 & 0.1194058 & 2.3996502 \\
\hline 0.5 & & 0.0542534 & 2.3835712 & - & - & - & - & 0.0542528 & 2.3835713 \\
\hline
\end{tabular}

heat flux, and $\mathrm{j}_{m}$ is the surface mass flux, which are given by

$$
\begin{aligned}
& \tau_{w x}=\left.\left(\left(\mu+\frac{1}{\beta b}\right) \frac{\partial u}{\partial y}-\frac{1}{6 \beta b^{3}}\left(\frac{\partial u}{\partial y}\right)^{3}\right)\right|_{y=0}, \quad \tau_{w z}=\left.\left(\left(\mu+\frac{1}{\beta b}\right) \frac{\partial w}{\partial y}-\frac{1}{6 \beta b^{3}}\left(\frac{\partial w}{\partial y}\right)^{3}\right)\right|_{y=0}, \\
& q_{w}=-\left.k\left(\frac{\partial T}{\partial y}\right)\right|_{y=0}, \quad j_{m}=-\left.D_{B}\left(\frac{\partial \mathrm{C}}{\partial y}\right)\right|_{y=0} .
\end{aligned}
$$

From equations (10), (11), (17), and (18), we obtain

$$
\begin{aligned}
C_{f x} \sqrt{\mathrm{Re}_{x}} & =(1+\varepsilon) f^{\prime \prime}(0)-\frac{\varepsilon \delta}{3}\left(f^{\prime \prime}(0)\right)^{3}, \quad C_{g z} \sqrt{\mathrm{Re}_{x}}=(1+\varepsilon) g^{\prime}(0)-\frac{\varepsilon \delta}{3}\left(g^{\prime}(0)\right)^{3}, \\
N u_{x}\left(\sqrt{\mathrm{Re}_{x}}\right)^{-1} & =-\theta^{\prime}(0), \quad S h_{x}\left(\sqrt{\mathrm{Re}_{x}}\right)^{-1}=-\phi^{\prime}(0) .
\end{aligned}
$$

\section{Method of Solution}

Equations (12)-(15) subject to the boundary conditions (16) are explained utilizing the spectral relaxation method. The spectral relaxation algorithm uses the thought of the GaussSeidel method to decouple the arrangement of overseeing equations (12)-(15). The strategy is created by assessing the linear terms at the current iteration level $r+1$ and nonlinear terms at the former iteration level $r$. The Chebyshev pseudospectral technique is used to unravel the decoupled equations. For the subtleties of the technique, interested readers can refer [3, 46-48].

Hence, to utilize the SRM, we start by decreasing the request for the momentum (equation (12)) from third to second order presenting the change $f^{\prime}=h$ so that $f^{\prime \prime}=h^{\prime}$ and $f^{\prime \prime \prime}=h^{\prime \prime}$.Along these lines, equations (12)-(15) become

$$
f^{\prime}=h
$$

$$
\begin{aligned}
& (1+\varepsilon) h^{\prime \prime}-(\varepsilon \delta) h^{\prime 2} h^{\prime \prime}-h^{2}+f h^{\prime}-\frac{M}{\left(\alpha_{e}^{2}+\beta_{e}^{2}\right)}\left(\alpha_{e} h+\beta_{e} g\right) \\
& +\lambda(\theta+N r \phi)-K h=0, \\
& (1+\varepsilon) g^{\prime \prime}-(\varepsilon \delta) g^{\prime 2} g^{\prime \prime}-h g+f g^{\prime}+\frac{M}{\left(\alpha_{e}^{2}+\beta_{e}^{2}\right)}\left(\beta_{e} h-\alpha_{e} g\right)-K g=0,
\end{aligned}
$$

$$
\begin{aligned}
& \frac{1}{\operatorname{Pr}} \theta^{\prime \prime}+f \theta^{\prime}-\gamma_{1}\left(f h \theta^{\prime}+f^{2} \theta^{\prime \prime}\right)+N b \theta^{\prime} \phi^{\prime}+N t \theta^{\prime 2} \\
& +\frac{M \cdot E c}{\left(\alpha_{e}^{2}+\beta_{e}^{2}\right)}\left(h^{2}+g^{2}\right)=0
\end{aligned}
$$




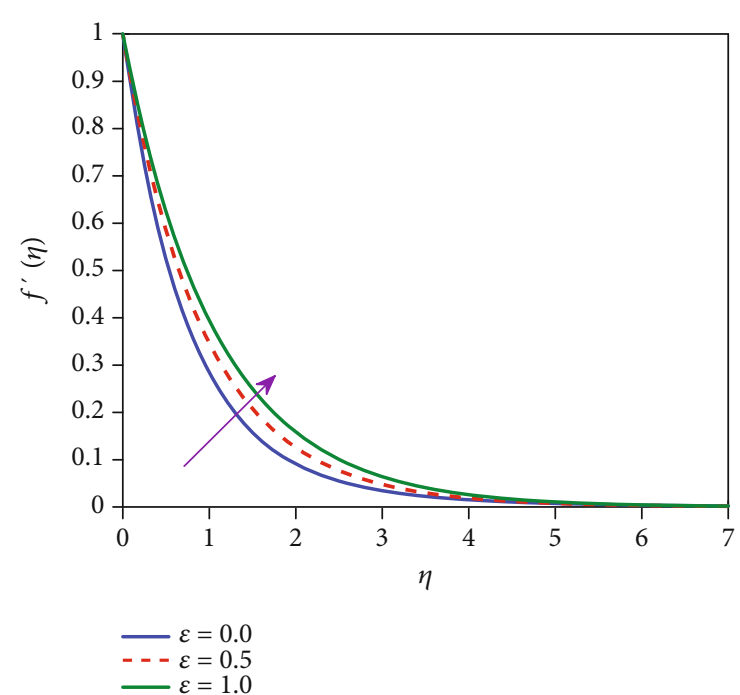

FIgURE 2: Effect of $\varepsilon$ on the primary velocity $f^{\prime}(\eta)$.

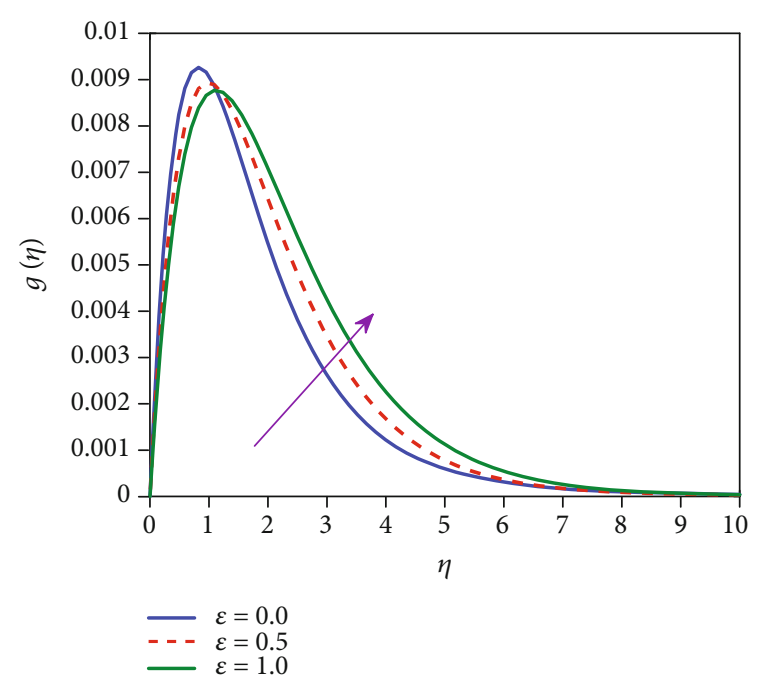

Figure 3: Effect of $\varepsilon$ on the secondary velocity $g(\eta)$.

$$
\phi^{\prime \prime}+\operatorname{Pr} . L e\left[f \phi^{\prime}-\gamma_{2}\left(f h \phi^{\prime}+f^{2} \phi^{\prime \prime}\right)\right]+\frac{N t}{N b} \theta^{\prime \prime}=0 .
$$

The transformed boundary conditions are

$$
\begin{aligned}
& f(0)=0, \quad h(0)=1, \quad g(0)=0, \quad \theta(0)=1, \quad \phi(0)=1, \\
& h \longrightarrow 0, \quad g \longrightarrow 0, \quad \theta \longrightarrow 0, \quad \phi \longrightarrow 0, \quad \text { as } \quad \eta \longrightarrow \infty .
\end{aligned}
$$

Executing the SRM to equations (20)-(25), we get the following iteration scheme:

$$
\begin{aligned}
& (1+\varepsilon) h_{r+1}^{\prime \prime}-(\varepsilon \delta) h_{r}^{\prime 2} h_{r}^{\prime \prime}-h_{r}^{2}+f_{r} h_{r+1}^{\prime} \\
& \quad-\frac{M}{\left(\alpha_{e}^{2}+\beta_{e}^{2}\right)}\left(\alpha_{e} h_{r+1}+\beta_{e} g_{r}\right)+\lambda\left(\theta_{r}+N r \phi_{r}\right)-K h_{r+1}=0
\end{aligned}
$$

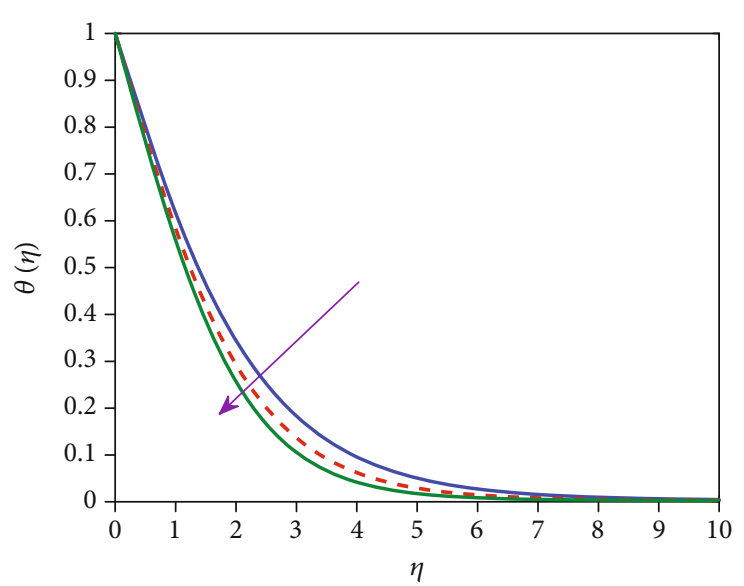

$$
\begin{aligned}
-\varepsilon & =0.0 \\
---\varepsilon & =0.5 \\
-\varepsilon & =1.0
\end{aligned}
$$

FIgURE 4: Effect of $\varepsilon$ on the temperature $\theta(\eta)$.

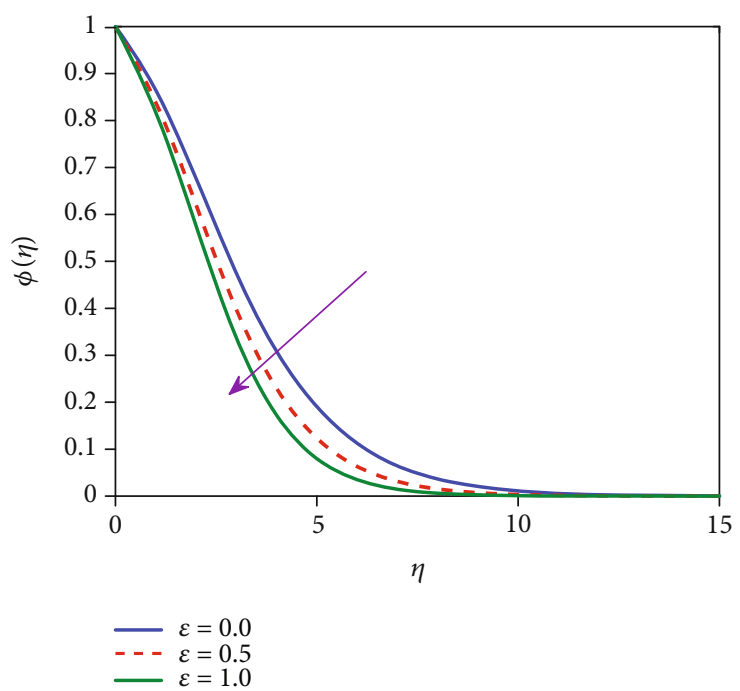

FIgURE 5: Effect of $\varepsilon$ on the concentration $\phi(\eta)$.

$$
f_{r+1}^{\prime}=h_{r+1}
$$

$$
\begin{aligned}
& (1+\varepsilon) g_{r+1}^{\prime \prime}-(\varepsilon \delta) g_{r}^{\prime 2} g_{r}^{\prime \prime}-h_{r+1} g_{r+1}+f_{r+1} g_{r+1}^{\prime} \\
& +\frac{M}{\left(\alpha_{e}^{2}+\beta_{e}^{2}\right)}\left(\beta_{e} h_{r+1}-\alpha_{e} g_{r+1}\right)-K g_{r+1}=0
\end{aligned}
$$

$$
\begin{aligned}
& \frac{1}{\operatorname{Pr}} \theta_{r+1}^{\prime \prime}+f_{r+1} \theta_{r+1}^{\prime}-\gamma_{1}\left(f_{r+1} h_{r+1} \theta_{r+1}^{\prime}+f_{r}^{2} \theta_{r+1}^{\prime \prime}\right)+N b \theta_{r+1}^{\prime} \phi_{r+1}^{\prime} \\
& \quad+N t \theta_{r}^{\prime 2}+\frac{M \cdot E c}{\left(\alpha_{e}^{2}+\beta_{e}^{2}\right)}\left(h_{r}^{2}+g_{r}^{2}\right)=0,
\end{aligned}
$$

$\phi_{r+1}^{\prime \prime}+\operatorname{Pr} \cdot L e\left[f_{r+1} \phi_{r+1}^{\prime}-\gamma_{2}\left(f_{r+1} h_{r+1} \phi_{r+1}^{\prime}+f_{r}^{2} \phi_{r+1}^{\prime \prime}\right)\right]+\frac{N t}{N b} \theta_{r+1}^{\prime \prime}=0$. 


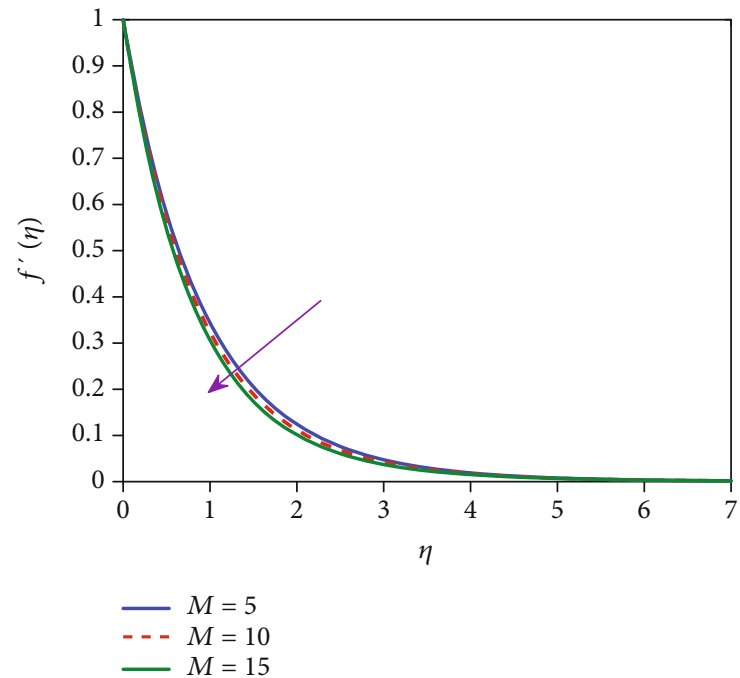

FIGURE 6: Effect of $M$ on the primary velocity $f^{\prime}(\eta)$.

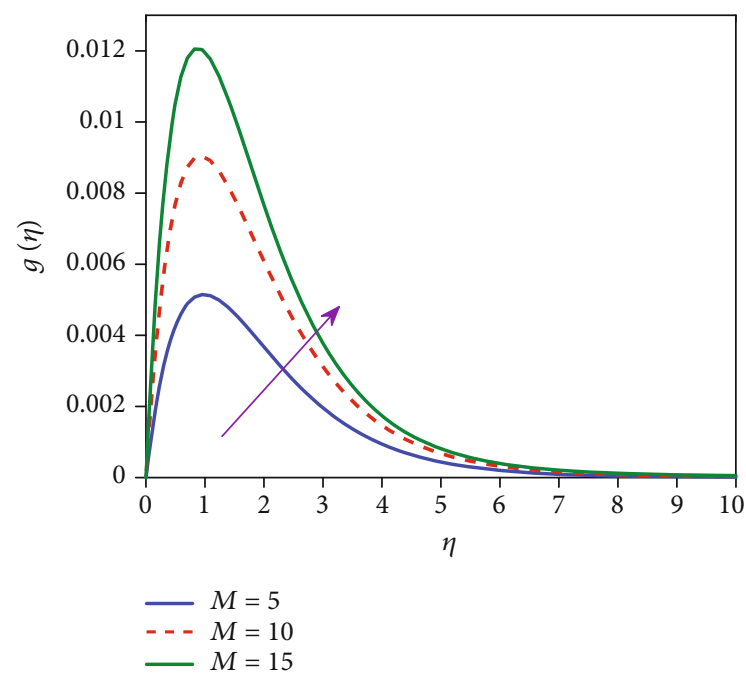

Figure 7: Effect of $M$ on the secondary velocity $g(\eta)$.

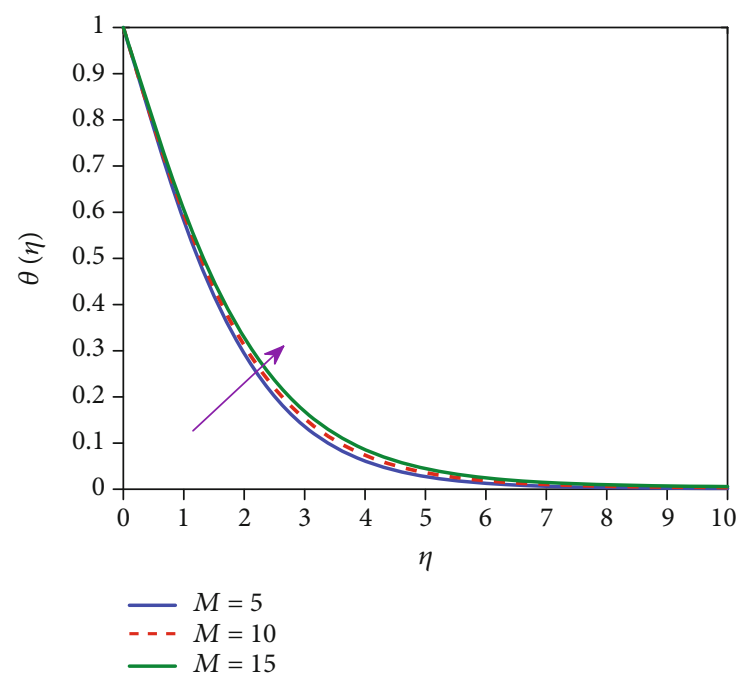

FIGURE 8: Effect of $M$ on the temperature $\theta(\eta)$.

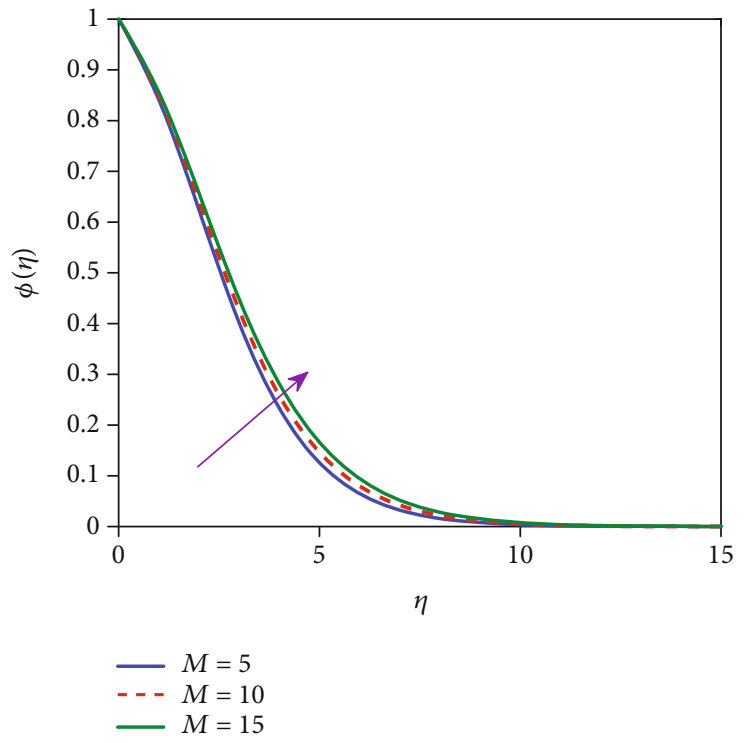

Figure 9: Effect of $M$ on the concentration $\phi(\eta)$.

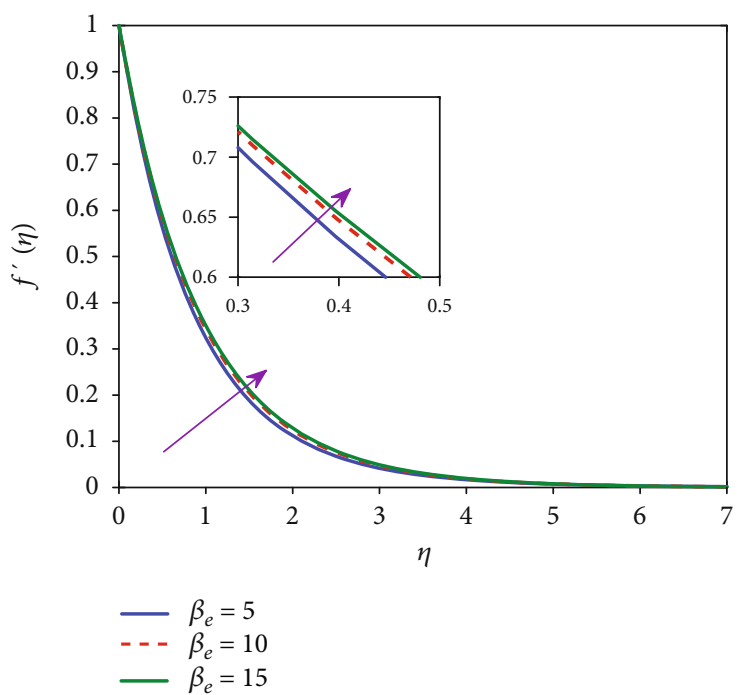

FIGURE 10: Effect of $\beta_{e}$ on the primary velocity $f^{\prime}(\eta)$.

The transformed boundary conditions are

$f_{r+1}(0)=0, \quad h_{r+1}(0)=1, \quad g_{r+1}(0)=0, \quad \theta_{r+1}(0)=1, \quad \phi_{r+1}(0)=1$, $h_{r+1} \longrightarrow 0, \quad g_{r+1} \longrightarrow 0, \quad \theta_{r+1} \longrightarrow 0, \quad \phi_{r+1} \longrightarrow 0, \quad$ as $\quad \eta \longrightarrow \infty$.

Actualizing the Chebyshev spectral collocation method to equations (26)-(30), we get the ensuing matrix equations:

$$
\begin{aligned}
& A_{1} \boldsymbol{h}_{r+1}=B_{1}, \quad h_{r+1}\left(\xi_{N}\right)=1, \quad h_{r+1}\left(\xi_{0}\right)=0, \\
& A_{2} f_{r+1}=B_{2}, \quad f_{r+1}\left(\xi_{N}\right)=0, \\
& A_{3} \boldsymbol{g}_{\mathrm{r}+1}=\mathrm{B}_{3}, \quad g_{r+1}\left(\xi_{N}\right)=0, \quad g_{r+1}\left(\xi_{0}\right)=0,
\end{aligned}
$$




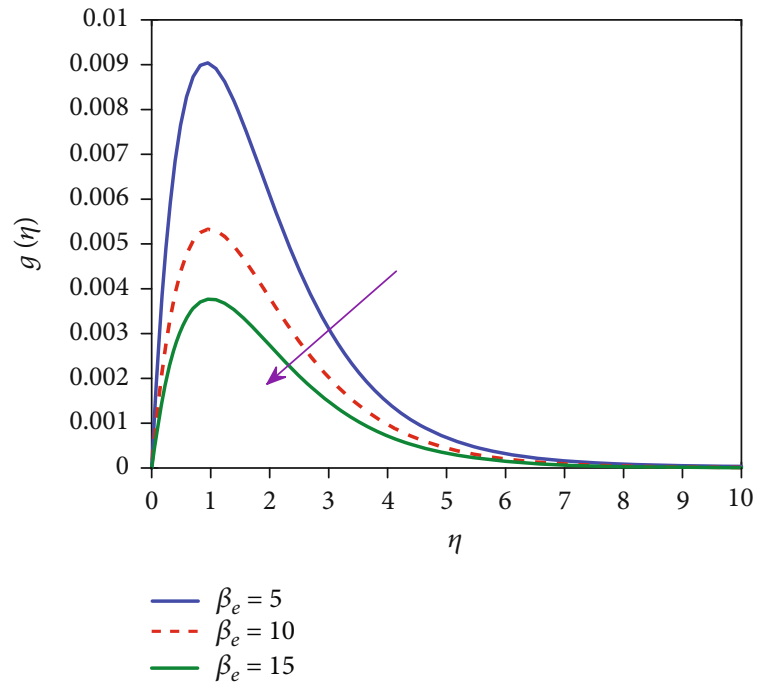

FIGURE 11: Effect of $\beta_{e}$ on the secondary velocity $g(\eta)$.

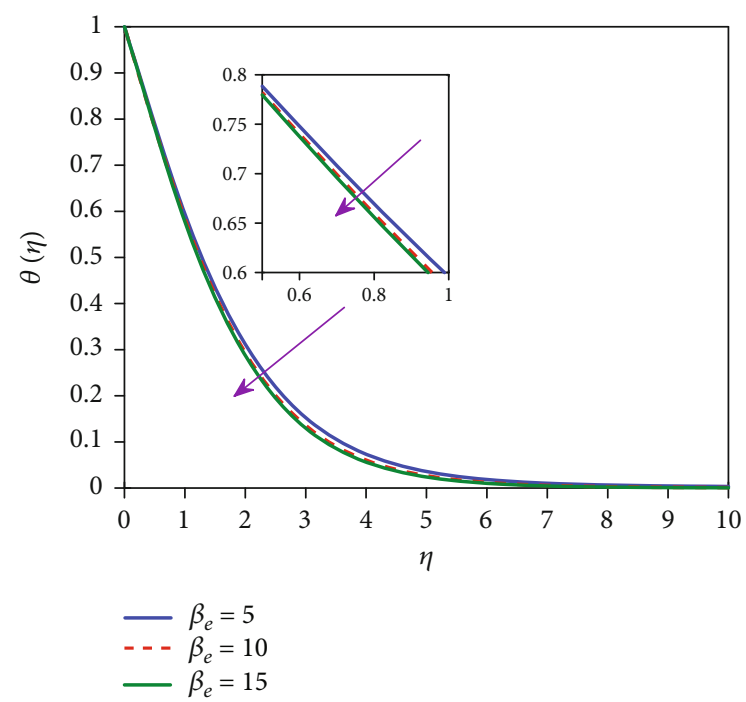

Figure 12: Effect of $\beta_{e}$ on the temperature $\theta(\eta)$.

$$
\begin{aligned}
& A_{4} \theta_{\mathrm{r}+1}=\mathrm{B}_{4}, \quad \theta_{r+1}\left(\xi_{N}\right)=1, \quad \theta_{r+1}\left(\xi_{0}\right)=0, \\
& A_{5} \phi_{\mathrm{r}+1}=\mathrm{B}_{5}, \quad \phi_{r+1}\left(\xi_{N}\right)=1, \quad \phi_{r+1}\left(\xi_{0}\right)=0,
\end{aligned}
$$

where

$$
\begin{aligned}
& A_{1}=(1+\varepsilon) \boldsymbol{D}^{2}+\operatorname{diag}\left(f_{r}\right) \boldsymbol{D}-\left(\frac{M \alpha_{e}}{\alpha_{e}^{2}+\beta_{e}^{2}}+K\right) \boldsymbol{I} \\
& \mathrm{B}_{1}=(\varepsilon \delta) h_{r}^{\prime 2} h_{r}^{\prime \prime}+h_{r}^{2}+\frac{M \beta_{e}}{\left(\alpha_{e}^{2}+\beta_{e}^{2}\right)} g_{r}-\lambda\left(\theta_{r}+N r \phi_{r}\right), \\
& A_{2}=\boldsymbol{D}, B_{2}=h_{r+1}, \\
& A_{3}=(1+\varepsilon) \boldsymbol{D}^{2}+\operatorname{diag}\left(f_{r+1}\right) \boldsymbol{D}-\operatorname{diag}\left(h_{r+1}+\frac{M \alpha_{e}}{\alpha_{e}^{2}+\beta_{e}^{2}}+K\right) \boldsymbol{I}
\end{aligned}
$$

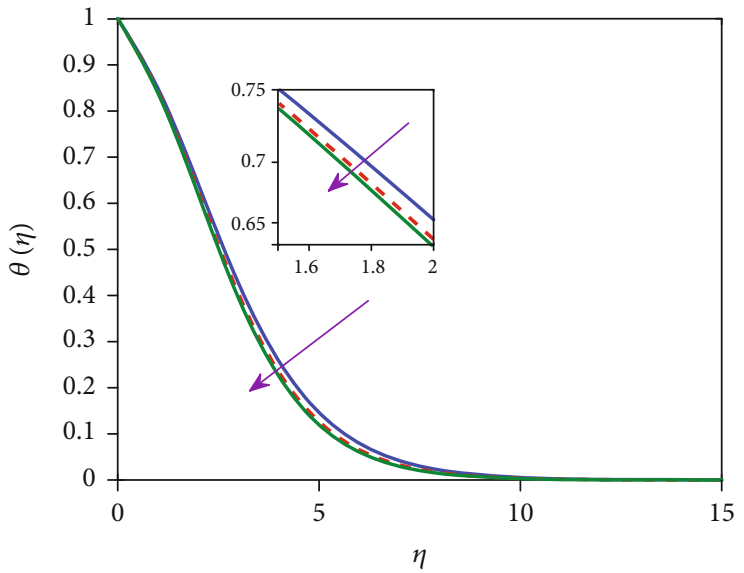

$$
\begin{aligned}
- & \beta_{e}=5 \\
--- & \beta_{e}=10 \\
- & \beta_{e}=15
\end{aligned}
$$

Figure 13: Effect of $\beta_{e}$ on the concentration $\phi(\eta)$.

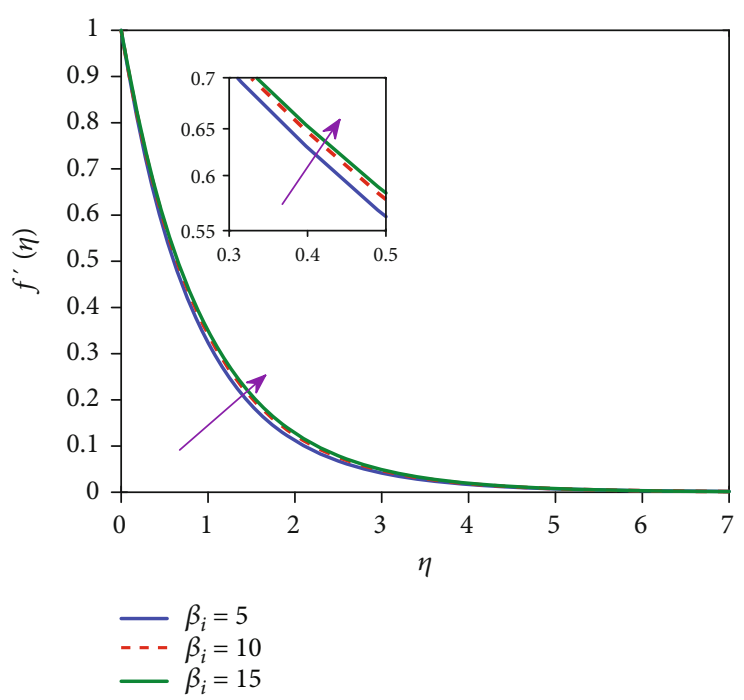

FIgURE 14: Effect of $\beta_{i}$ on the primary velocity $f^{\prime}(\eta)$.

$$
\begin{aligned}
& B_{3}=(\varepsilon \delta) g_{r}^{\prime 2} g_{r}^{\prime \prime}-\frac{M \beta_{e}}{\alpha_{e}^{2}+\beta_{e}^{2}} h_{r+1}, \\
& A_{4}=\operatorname{diag}\left(\frac{1}{\operatorname{Pr}}-\gamma_{1} f_{r}^{2}\right) \boldsymbol{D}^{2}+\operatorname{diag}\left(f_{r+1}-\gamma_{1} f_{r+1} h_{r+1}+N b \phi_{r}^{\prime}\right) \boldsymbol{D}, \\
& B_{4}=-\frac{M \cdot E c}{\left(\alpha_{e}^{2}+\beta_{e}^{2}\right)}\left(h_{r}^{2}+g_{r}^{2}\right)-N t \theta_{r}^{\prime 2}, \\
& A_{5}=\operatorname{diag}\left(1-\operatorname{PrLe\gamma _{2}} f_{r}^{2}\right) \boldsymbol{D}^{2}+\operatorname{PrLe} \operatorname{diag}\left(f_{r+1}-\gamma_{2} f_{r+1} h_{r+1}\right) \boldsymbol{D}, \\
& B_{5}=-\frac{N t}{N b} \theta_{r+1}^{\prime \prime} .
\end{aligned}
$$

Here, $\boldsymbol{D}=2 D / \eta_{\infty}$ where $D$ is the Chebyshev differentiation matrix (Trefethen [50]), $\eta_{\infty}$ is a limited length which is adequately big so that we can simply incorporate the condition at perpetuity in this point, $\boldsymbol{I}$ and diag [.] are the identity and diagonal matrices of order $(N+1) \times(N+1), N$ is the 


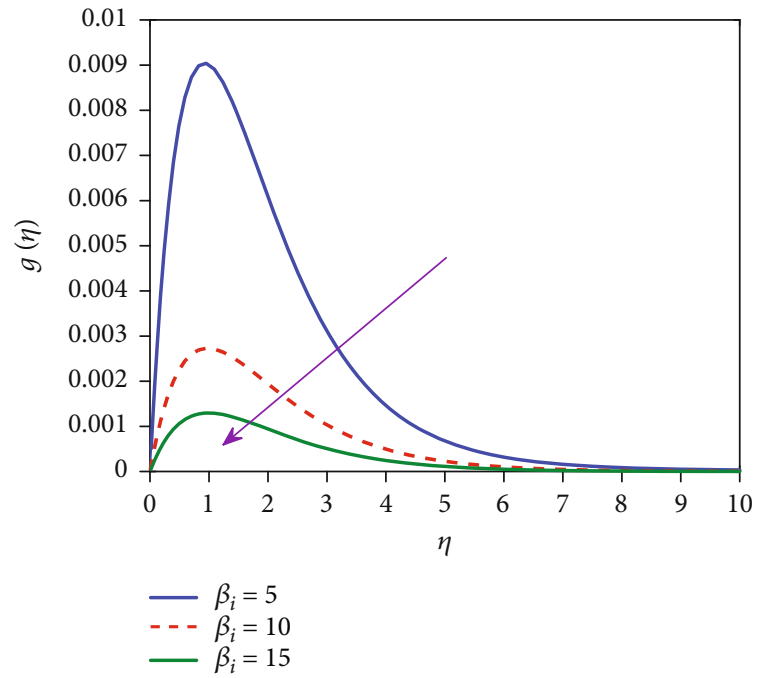

Figure 15: Effect of $\beta_{i}$ on the secondary velocity $g(\eta)$.

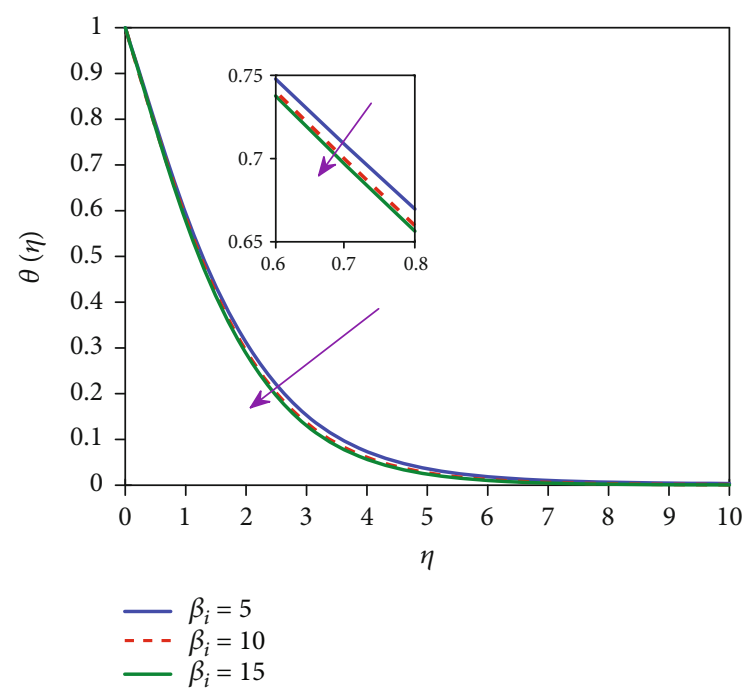

FIGURE 16: Effect of $\beta_{i}$ on the temperature $\theta(\eta)$.

number of grid points, and $\boldsymbol{f}, \boldsymbol{h}, \boldsymbol{g}, \boldsymbol{\theta}$ and $\boldsymbol{\phi}$ are, respectively, the values of $f, h, g, \theta$, and $\phi$, when assessed at the grid points.

The decoupled equations (32)-(36) can be solved separately by picking appropriate introductory suppositions:

$$
f_{0}(\eta)=1-\mathrm{e}^{-\eta}, g_{0}(\eta)=\eta \mathrm{e}^{-\eta}, \theta_{0}(\eta)=\mathrm{e}^{-\eta}=\phi_{0}(\eta)
$$

\section{Results and Discussions}

The nonlinear differential equations (12)-(15) with the limit conditions (16) have been explained numerically via the spectral relaxation method, and consequences of the numerical calculations for the velocity, temperature, and concentration profiles as well as skin friction coefficients, local Nusselt number, and Sherwood number have been obtained for different input parameters and presented through graphs and tables. Also, we contrasted our outcomes and those of the current writing as appeared in Table 1, and the outcomes give

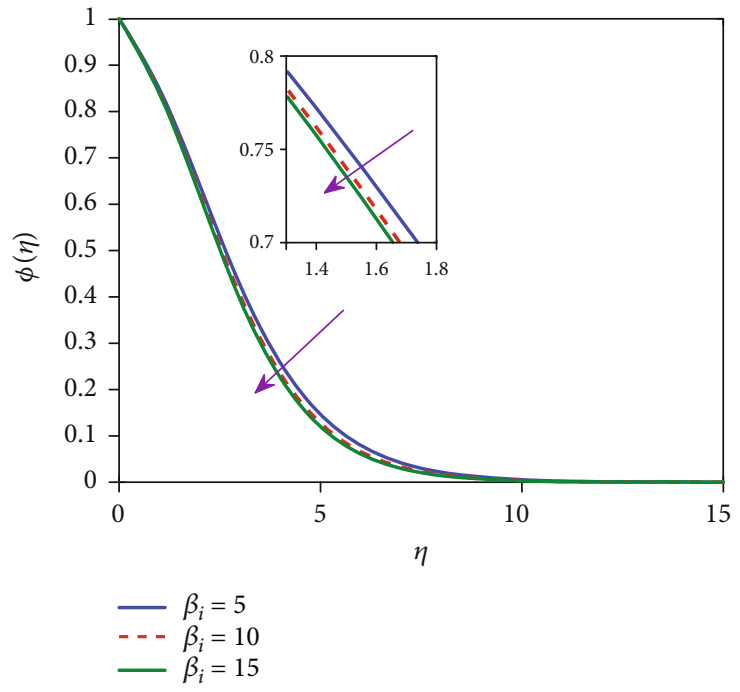

Figure 17: Effect of $\beta_{i}$ on the concentration $\phi(\eta)$.

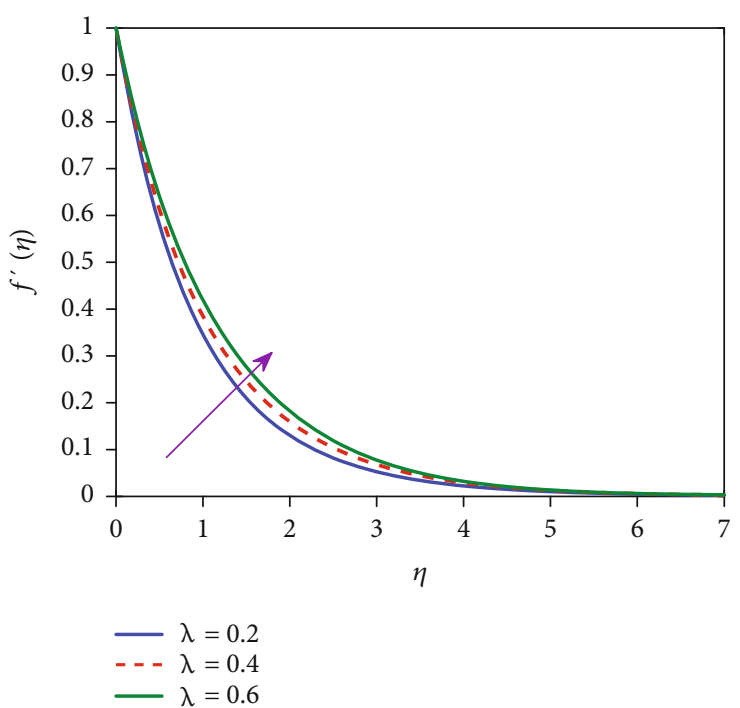

Figure 18: Effect of $\lambda$ on the primary velocity $f^{\prime}(\eta)$.

a generally excellent understanding which gives a certainty of our current numerical outcomes. In this study, for our numerical computations, we fixed the numerical values of the physical parameters as $=0.1, L e=1.0, M=10.0, \beta_{e}=$ $\beta_{i}=5.0, \operatorname{Pr}=0.72, K=0.5, N r=N b=N t=0.1, E c=0.02$, $\delta=0.2$, and $\varepsilon=\gamma_{1}=\gamma_{2}=0.3$ unless otherwise stated.

Figures 2-5 reveal the impact of the Eyring-Powell fluid parameter $(\varepsilon)$ on the primary velocity, secondary velocity, temperature, and concentration distributions, respectively. By expanding the liquid parameter $\varepsilon$, the primary velocity profile rises and on the contrary, the temperature and concentration profiles diminish. Because the fluid parameter $\varepsilon$ $(=1 / \mu \beta \mathrm{b})$ has an inverse relation with viscosity of fluid $(\mu)$; thus, fluid turns out to be less viscous for bigger values of $\varepsilon$ which upsurges fluid velocity. Moreover, the secondary velocity profile reduces near the surface and enhances far off from the surface. This is possibly due to the fact that the 


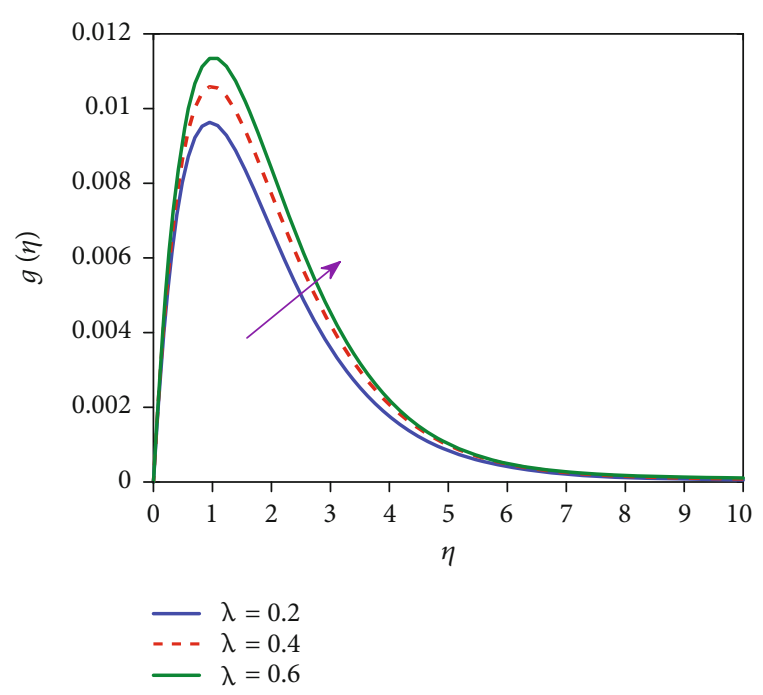

Figure 19: Effect of $\lambda$ on the secondary velocity $g(\eta)$.

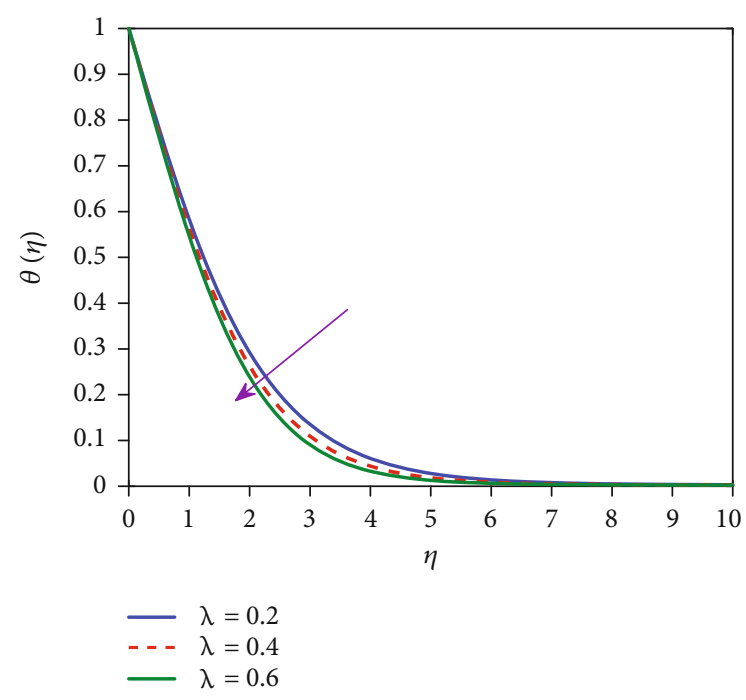

Figure 20: Effect of $\lambda$ on the temperature $\theta(\eta)$.

viscosity can be varied as the Eyring-Powell fluid parameter varies resulting in an increasing viscous force as a result decreasing its velocity up to some maximum value, and then the velocity starts to increase due to decrease in viscosity. The optimum value is obtained around the point $\approx 1$.

Figures 6-9 divulge the impact of magnetic field parameter $\mathrm{M}$ on primary velocity, secondary velocity, temperature, and concentration profiles, respectively. The transverse magnetite field which is applied vertical to the direction of flow provides a resistive force known as the Lorentz force. Physically, the Lorentz force opposes the flow of nanofluid; hence, the velocity diminishes. Moreover, this force tends to hinder the movement of the liquid in the boundary layer. This assists with declining the primary velocity profile and upgrades the secondary velocity, thermal, and concentration profiles as $\mathrm{M}$ increments as appeared in Figures 6-9. It is additionally seen that an augmentation in $\mathrm{M}$ prompts a lower in primary velocity boundary layer thickness and superior in thermal

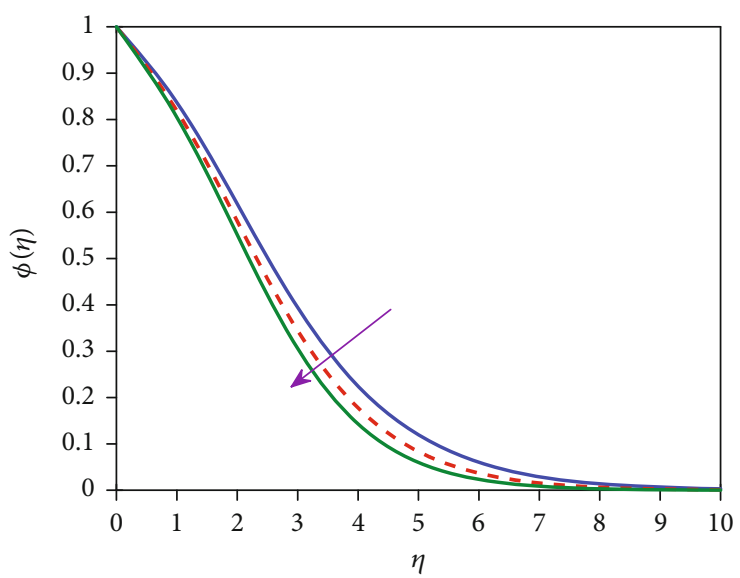

$$
\begin{aligned}
-\lambda & =0.2 \\
---\lambda & =0.4 \\
-\lambda & =0.6
\end{aligned}
$$

Figure 21: Effect of $\lambda$ on the concentration $\phi(\eta)$.

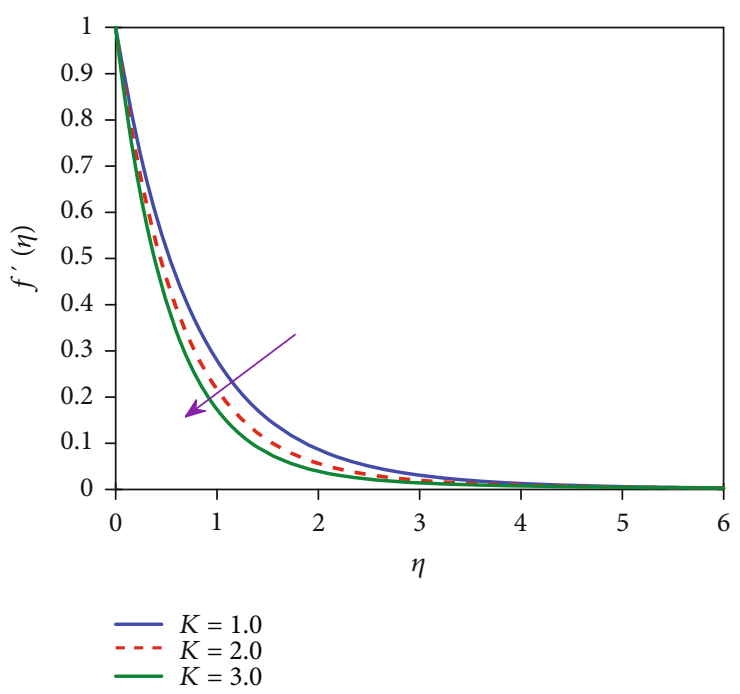

FIGURE 22: Effect of $K$ on the primary velocity $f^{\prime}(\eta)$.

and concentration boundary layer thicknesses, though the secondary velocity boundary layer thickness diminishes near the surface and upsurges far off from the surface.

Figures 10-13 illustrate the impact of the Hall parameter $\beta_{e}$ on primary velocity, secondary velocity, temperature, and concentration profiles, respectively. It is seen that the primary velocity profile increments with an expansion in $\beta_{e}$, though the contrary condition is watched for secondary velocity, thermal, and concentration profiles. Besides, as the Hall parameter $\beta_{e}$ enlarges, the primary velocity boundary layer thickness upgrades, and thermal and concentration boundary layer thicknesses lessen, while the secondary velocity boundary layer thickness improves near the surface and diminishes distant from the surface. Physically, the consideration of Hall parameter decreases the efficient conductivity and subsequently drops the magnetic resistive force. Thus, the Joule heating impact is condensed, and quantity of heat 


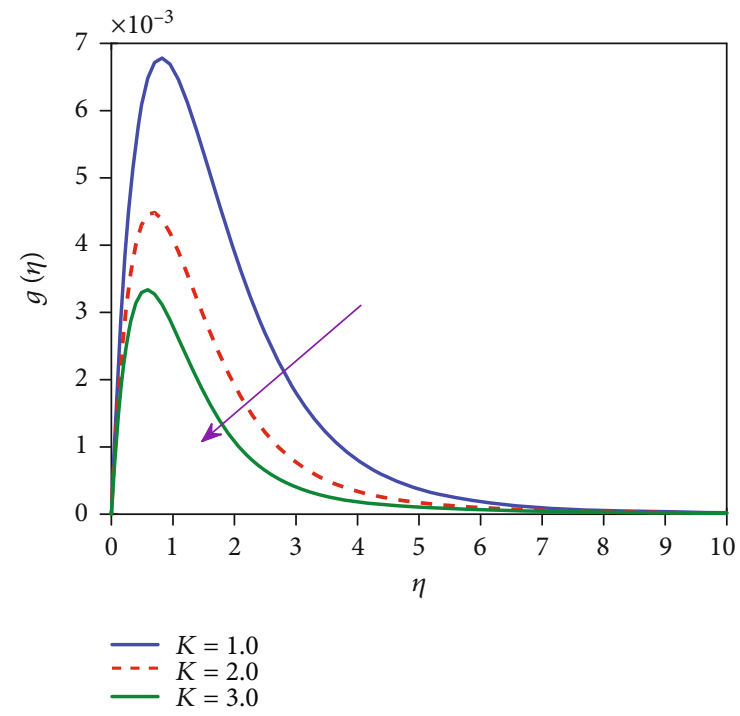

Figure 23: Effect of $K$ on the secondary velocity $g(\eta)$.

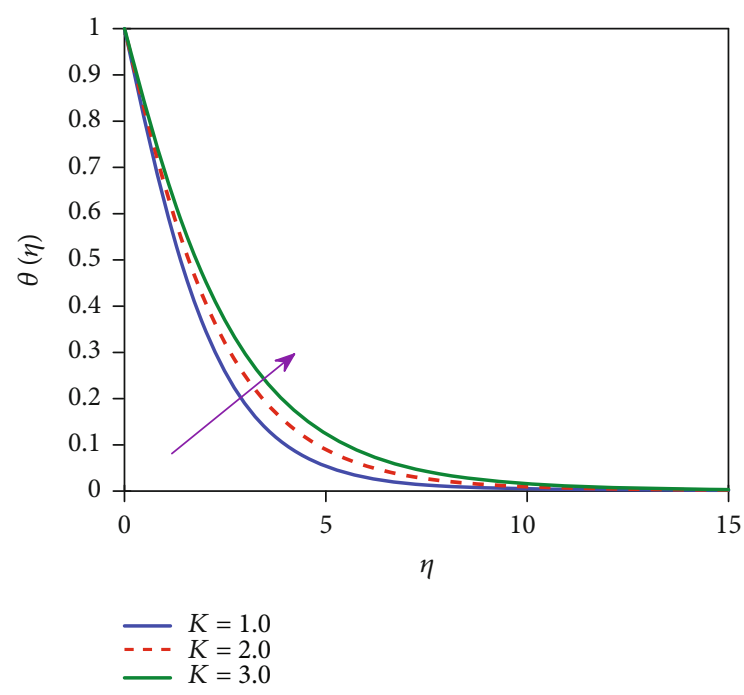

Figure 24: Effect of $K$ on the temperature $\theta(\eta)$.

owing to ohmic dissipation can be abridged by utilizing partially ionized fluid presented to magnetic field. The comparable conduct is seen with expanding ion slip parameter $\beta_{i}$ as revealed in Figures 14-17. Physically, the expansion in $\beta_{i}$ improves the efficient conductivity and therefore diminishes the damping force on the dimensionless velocity, and hence the dimensionless velocity expands.

Figures 18-21 portray the impact of blended convection parameter $\lambda$ on primary velocity, secondary velocity, temperature, and concentration profiles, respectively. It is deduced that as $\lambda$ upgrades, the primary velocity and secondary velocity profiles ascend. In contrast, the temperature and concentration profiles reduce. These outcomes physically hold since upgrade in blended convection parameter causes upsurge in buoyancy forces which speed up fluid motion.

Figures 22-25 outline the impact of penetrability parameter $K$ on the primary velocity, secondary velocity, thermal,

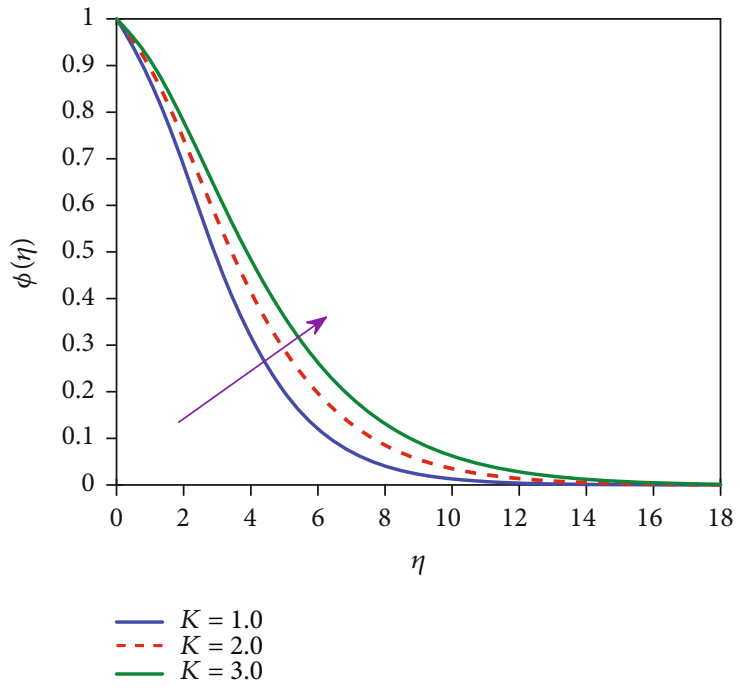

Figure 25: Effect of $K$ on the concentration $\phi(\eta)$.

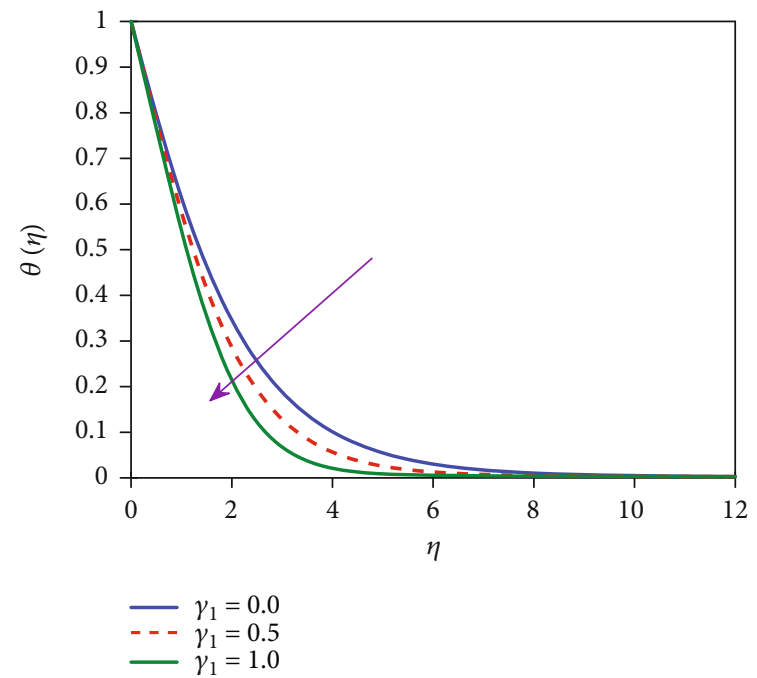

Figure 26: Effect of $\gamma_{1}$ on the temperature $\theta(\eta)$.

and concentration distributions, respectively. It is obvious from the figures that as $K$ augments, both primary and secondary velocity profiles decline while the opposite condition is watched for thermal and concentration profiles. Physically, the porousness builds obstruction of the permeable medium which will in general, diminish the fluid velocity. It is normal that an expansion in the porousness of the permeable medium prompts the ascent in the flow of fluid through it. When the outlets of the permeable medium become big, the obstruction of the medium might be ignored.

Figure 26 proves the impact of the thermal relaxation parameter $\gamma_{1}$ on temperature distribution. It uncovers that the fluid temperature and energy boundary layer decrease with expanding value of $\gamma_{1}$. Physically, thermal relaxation time is the time required by the fluid particles to move heat energy to its neighboring particles. Subsequently, as $\gamma_{1}$ ascends, the material particles need additional time to transfer heat to its adjoining particles, and this prompts less 


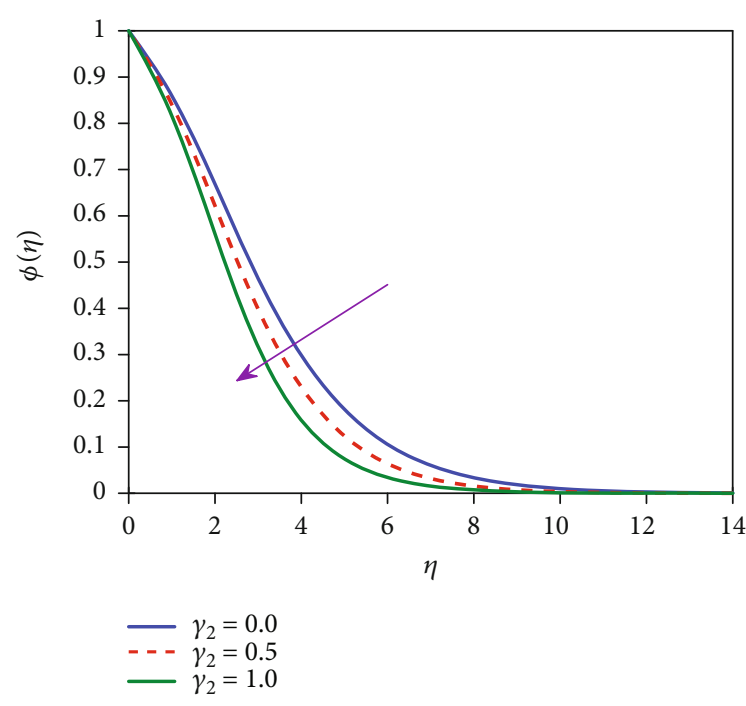

Figure 27: Effect of $\gamma_{2}$ on the concentration $\phi(\eta)$.

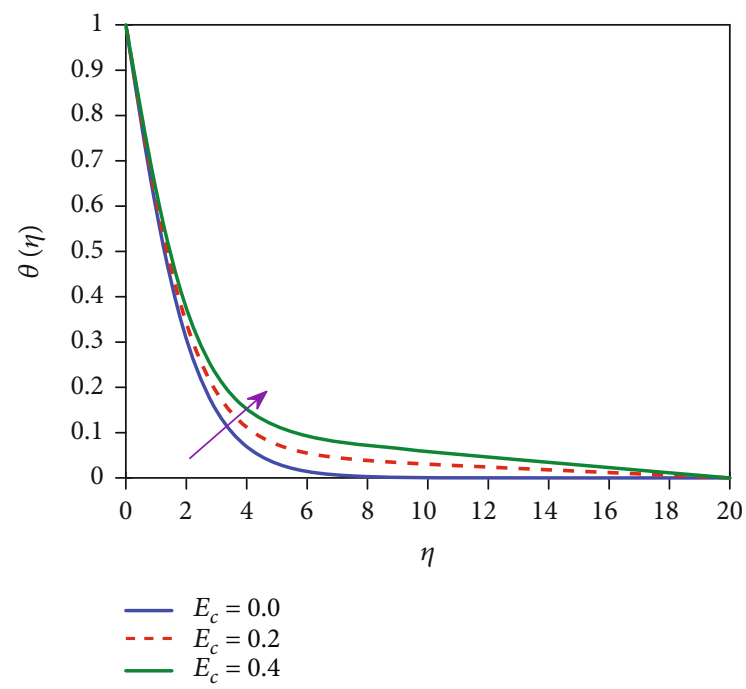

Figure 28: Effect of $E c$ on the temperature $\theta(\eta)$.

transport of heat from the surface to the fluid. Similarly, the concentration relaxation parameter $\gamma_{2}$ emerges from the relaxation time of mass flux. Less relaxation time of mass flux prompts superior concentration. and the fluid with increasingly mass flux relaxation time brings about smaller concentration. Therefore, increasingly mass flux relaxation time happens owing to the bigger value of $\gamma_{2}$ which diminishes the concentration profile as revealed in Figure 27.

Figure 28 depicts the impact of Eckert number $E c$ on the temperature profile. It is seen that the temperature profile upsurges with an expansion in Ec. Physically, by expanding the Eckert number, the heat energy is hoarded in the fluid as a result of the frictional or drag forces. Accordingly, the fluid temperature field enhances.

Figure 29 demonstrates the effect of Lewis number Le on the concentration profile $\phi(\eta)$. By expanding $L e$, the concentration and the boundary layer thicknesses decline. This is because of the way that the mass transfer rate enhances by

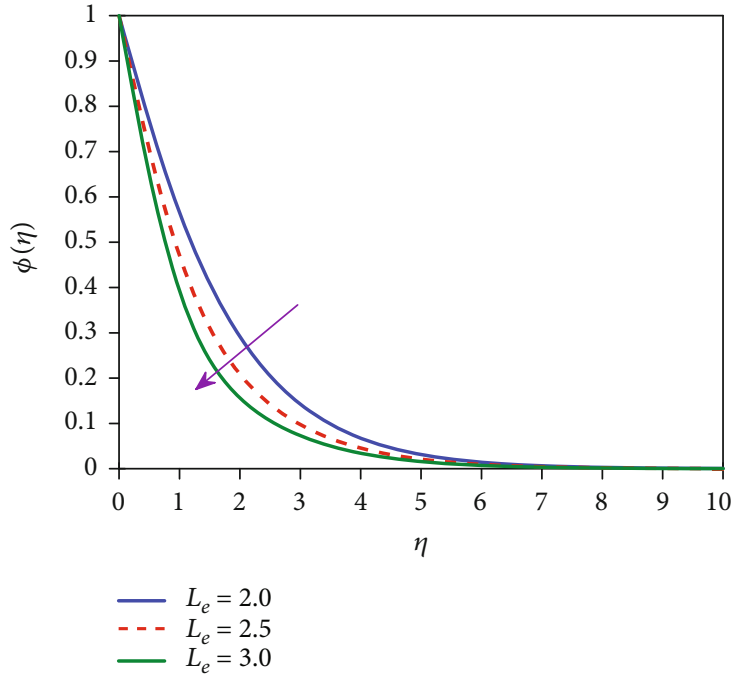

Figure 29: Effect of Le on the concentration $\phi(\eta)$.

expanding Le which improves the volume fraction of nanoparticles.

Table 2 specifies the impact of physical parameters on the skin fraction coefficients, local Nusselt, and Sherwood numbers. The primary skin friction coefficient $-\sqrt{\operatorname{Re}} C_{f x}$ augments as magnetic parameter $M$, Eyring-Powell fluid parameter $\varepsilon$, thermal relaxation parameter $\gamma_{1}$, concentration relaxation parameter $\gamma_{2}$, and permeability parameter $K$ rise and decreases for increasing values of mixed convection parameter $\lambda$, Hall parameter $\beta_{e}$, ion slip parameter $\beta_{i}$, and Eckert number $E c$. Also, it is observed that the secondary skin friction coefficient $\sqrt{\operatorname{Re}} C_{g z}$ amplifies as magnetic parameter $M$, mixed convection parameter $\lambda$, and Eyring-Powell fluid parameter $\varepsilon$ increase and declines as Hall parameter $\beta_{e}$, ion slip parameter $\beta_{i}$, thermal relaxation parameter $\gamma_{1}$, concentration relaxation parameter $\gamma_{2}$, and permeability parameter $K$ increase, while it remains constant as the Eckert number $E c$ enhances. Similarly, both the local Nusselt number $-\theta^{\prime}(0)$ and Sherwood number $-\phi^{\prime}(0)$ enhance for larger values of mixed convection parameter $\lambda$, Hall parameter $\beta_{e}$, ion slip parameter $\beta_{i}$, and Eyring-Powell fluid parameter $\varepsilon$ and decrease for larger values of magnetic parameter $M$ and permeability parameter $K$. Further, opposite behaviors of the local Nusselt number $-\theta^{\prime}(0)$ and the local Sherwood number $-\phi^{\prime}(0)$ are observed for increasing values of thermal relaxation parameter $\gamma_{1}$, concentration relaxation parameter $\gamma_{2}$, and Eckert number $E c$.

\section{Conclusions}

This paper looks at the mixed convection stream of EyringPowell nanofluid with the Cattaneo-Christov heat and mass flux model over linearly stretching sheet through a permeable medium. The impacts of Hall and ion slip, permeability, and Joule heating are also considered. The governing nonlinear partial differential equations are changed into joined nonlinear 
TABLE 2: Numerical values of the skin friction coefficients $\sqrt{\operatorname{Re}} C_{f x}=(1+\varepsilon) f^{\prime \prime}(0)-(\varepsilon \delta / 3)\left(f^{\prime \prime}(0)\right)^{3}$ and $\sqrt{\operatorname{Re}} C_{g z}=(1+\varepsilon) g^{\prime}(0)-(\varepsilon \delta / 3)$ $\left(g^{\prime}(0)\right)^{3}$, local Nusselt number $-\theta^{\prime}(0)$, and local Sherwood number $-\phi^{\prime}(0)$ for various values of $M, \lambda, \beta_{e}, \beta_{i}, \varepsilon, \gamma_{1}, \gamma_{2}, K$, and $E c$ when $N$ $r=1.0, \operatorname{Pr}=0.72, N b=0.2, N t=0.1, L e=1$, and $\delta=10$ are fixed.

\begin{tabular}{|c|c|c|c|c|c|c|c|c|c|c|c|c|}
\hline$M$ & $\lambda$ & $\beta_{e}$ & $\beta_{i}$ & $\varepsilon$ & $\gamma_{1}$ & $\gamma_{2}$ & $K$ & $E c$ & $-\sqrt{\operatorname{Re}} C_{f x}$ & $\sqrt{\operatorname{Re}} C_{g z}$ & $-\theta^{\prime}(0)$ & $-\phi^{\prime}(0)$ \\
\hline 5.0 & 0.5 & 5.0 & 5.0 & 0.3 & 0.5 & 0.5 & 2.0 & 0.02 & 1.47604 & 0.01432 & 0.43751 & 0.33050 \\
\hline 10.0 & & & & & & & & & 1.53447 & 0.02757 & 0.43011 & 0.32389 \\
\hline 15.0 & & & & & & & & & 1.59039 & 0.03987 & 0.42296 & 0.31755 \\
\hline \multirow[t]{24}{*}{10.0} & 0.2 & & & & & & & & 1.77436 & 0.02470 & 0.37874 & 0.27471 \\
\hline & 0.4 & & & & & & & & 1.61585 & 0.02671 & 0.41540 & 0.30992 \\
\hline & 0.6 & & & & & & & & 1.45292 & 0.02836 & 0.44328 & 0.33631 \\
\hline & 0.5 & 5.0 & & & & & & & 1.53447 & 0.02757 & 0.43011 & 0.32389 \\
\hline & & 10.0 & & & & & & & 1.47713 & 0.01485 & 0.43740 & 0.33037 \\
\hline & & 15.0 & & & & & & & 1.45699 & 0.01016 & 0.43993 & 0.33265 \\
\hline & & 5.0 & 5.0 & & & & & & 1.53447 & 0.02757 & 0.43011 & 0.32389 \\
\hline & & & 10.0 & & & & & & 1.47883 & 0.00763 & 0.43719 & 0.33018 \\
\hline & & & 15.0 & & & & & & 1.45839 & 0.00350 & 0.43976 & 0.33249 \\
\hline & & & 5.0 & 0.0 & & & & & 1.38279 & 0.02496 & 0.42130 & 0.31614 \\
\hline & & & & 0.5 & & & & & 1.63596 & 0.02914 & 0.43581 & 0.32901 \\
\hline & & & & 1.0 & & & & & 1.88555 & 0.03270 & 0.44940 & 0.34156 \\
\hline & & & & 0.3 & 0.0 & & & & 1.53132 & 0.02769 & 0.40768 & 0.33589 \\
\hline & & & & & 0.5 & & & & 1.53447 & 0.02757 & 0.43011 & 0.32389 \\
\hline & & & & & 1.0 & & & & 1.53813 & 0.02743 & 0.45628 & 0.31008 \\
\hline & & & & & 0.5 & 0.0 & & & 1.53050 & 0.02771 & 0.43601 & 0.30351 \\
\hline & & & & & & 0.5 & & & 1.53447 & 0.02757 & 0.43011 & 0.32389 \\
\hline & & & & & & 1.0 & & & 1.53912 & 0.02740 & 0.42355 & 0.34772 \\
\hline & & & & & & 0.5 & 1.0 & & 1.18778 & 0.03474 & 0.47314 & 0.36359 \\
\hline & & & & & & & 2.0 & & 1.53447 & 0.02757 & 0.43011 & 0.32389 \\
\hline & & & & & & & 3.0 & & 1.79830 & 0.02303 & 0.39431 & 0.29262 \\
\hline & & & & & & & 2.0 & 0.0 & 1.53447 & 0.02757 & 0.43019 & 0.32385 \\
\hline & & & & & & & & 0.1 & 1.53446 & 0.02757 & 0.42980 & 0.32404 \\
\hline & & & & & & & & 0.2 & 1.53444 & 0.02757 & 0.42941 & 0.32422 \\
\hline
\end{tabular}

ordinary differential equations utilizing similitude transformations. These nonlinear ordinary differential equations subject to boundary conditions are handled numerically by means of SRM. Numerical results are presented for different parameters of interest, and results are discussed with the assistance of graphs and tables. The study has a momentous application in the material processing technology. From the introduced investigation, the accompanying ends were drawn:

(i) Primary velocity profile upsurges by increasing the values of Eyring-Powell fluid parameter $\varepsilon$, Hall parameter $\beta_{e}$, ion slip parameter $\beta_{i}$, and mixed convection parameter $\lambda$, while opposite condition is noticed for mounting the values of magnetic field parameter $M$ and porosity parameter $K$

(ii) Secondary velocity profile increases by increasing the values of Eyring-Powell fluid parameter $\varepsilon$ and magnetic field parameter $M$, while reverse behavior is observed for increasing values of Hall parameter $\beta_{e}$, ion slip parameter $\beta_{i}$, mixed convection parameter $\lambda$, and porosity parameter $K$

(iii) Both temperature and concentration profiles enhance with increasing values of magnetic field parameter $M$ and porosity parameter $K$, whereas reverse trend is noticed for increasing values of Eyring-Powell fluid parameter $\varepsilon$, Hall parameter $\beta_{e}$, ion slip parameter $\beta_{i}$, mixed convection parameter $\lambda$, thermal relaxation parameter $\gamma_{1}$, and concentration relaxation parameter $\gamma_{2}$. Moreover, temperature profile increases as Eckert number $E c$ increases, while concentration profile decreases as Lewis number $L e$ increases

(iv) Both skin friction coefficients increase with increasing values of magnetic field parameter $M$ and Eyring-Powell fluid parameter $\varepsilon$ and decrease with increasing values of Hall parameter $\beta_{e}$ and ion slip parameter $\beta_{i}$. On the contrary, opposite behavior is observed for the primary and secondary skin friction 
coefficients as mixed convection parameter $\lambda$, porosity parameter $K$, thermal relaxation parameter $\gamma_{1}$, and concentration relaxation parameter $\gamma_{2}$ upsurges. An increase in Eckert number $E c$ shows insignificant change in the primary skin friction coefficient, while the secondary skin friction coefficient remains constant

(v) Both local Nusselt and Sherwood numbers increase by enhancing the values of mixed convection parameter $\lambda$, Hall parameter $\beta_{e}$, ion slip parameter $\beta_{i}$, and Eyring-Powell fluid parameter $\varepsilon$, while opposite behavior is noticed for increasing values of magnetic field parameter $M$ and permeability parameter $K$. On the contrary, opposite behavior is observed for local Nusselt and Sherwood numbers as Eckert number $E c$, thermal relaxation parameter $\gamma_{1}$, and concentration relaxation parameter $\gamma_{2}$ increase

\section{Nomenclature}

$\begin{array}{ll}x, y, z: & \text { Cartesian coordinates }[\mathrm{m}] \\ u, v, w: & \text { Velocity components }[\mathrm{m} / \mathrm{s}] \\ U_{w}: & \text { Velocity of the stretching sheet }[\mathrm{m} / \mathrm{s}] \\ B_{0}: & \text { Constant magnetic field }\left[\mathrm{Wb} / \mathrm{m}^{2}\right] \\ \mathrm{g}: & \text { Acceleration due to gravity }\left[\mathrm{m} / \mathrm{s}^{2}\right] \\ T: & \text { Fluid temperature }[K] \\ T_{w}: & \text { Surface temperature }[\mathrm{K}] \\ T_{\infty}: & \text { Ambient temperature }[\mathrm{K}] \\ \mathrm{C}^{2} & \text { Concentration of fluid }\left[\mathrm{kg} / \mathrm{m}^{3}\right] \\ \mathrm{C}_{w}: & \text { Surface concentration }\left[\mathrm{kg} / \mathrm{m}^{3}\right] \\ \mathrm{C}_{\infty}: & \text { Ambient concentration }\left[\mathrm{kg} / \mathrm{m}^{3}\right] \\ M: & \text { Magnetic field parameter } \\ G r_{x}: & \text { Local Grashof number } \\ \mathrm{Re}_{x}: & \text { Local Reynolds number } \\ N r: & \text { Buoyancy ratio } \\ K: & \text { Permeability parameter } \\ \mathrm{Pr}: & \text { Prandtl number } \\ \mathrm{Nb}: & \text { Brownian motion parameter } \\ \mathrm{Nt}: & \text { Thermophoresis parameter } \\ E c: & \text { Eckert number } \\ L e: & \text { Lewis number } \\ C_{f x}: & \text { Skin friction coefficient in } x \text {-direction } \\ C_{g z}: & \text { Skin friction coefficient in } z \text {-direction } \\ N u_{x}: & \text { Local Nusselt number } \\ S h_{x}: & \text { Local Sherwood number } \\ f, g: & \text { Dimensionless stream functions }\end{array}$

\section{Greek letters}

$\rho: \quad$ Density of fluid $\left[\mathrm{kg} / \mathrm{m}^{3}\right]$

$v$ : Kinematic viscosity of the fluid $\left[\mathrm{m}^{2} / \mathrm{s}\right]$

$\varepsilon, \delta$ : Eyring-Powell fluid parameters

$\lambda$ : $\quad$ Mixed convection parameter

$\alpha$ : Thermal diffusivity $\left[\mathrm{m}^{2} / \mathrm{s}\right]$

$\kappa: \quad$ Thermal conductivity $[\mathrm{W} /(\mathrm{mK})]$

$\sigma: \quad$ Electrical conductivity $\left[\mathrm{m}^{2} / \mathrm{s}\right]$

$\beta_{e}$ : Hall parameter

$\beta_{i}$ : Ion slip parameter
$\beta_{T}$ : Coefficient of thermal expansion $[1 / K]$

$\beta_{C}$ : Coefficient of concentration expansion $[1 / K]$

$\psi: \quad$ Stream function

$\eta$ : Dimensionless similarity variable

$\theta: \quad$ Dimensionless temperature

$\phi$ : Dimensionless concentration

$\gamma_{1}$ : Thermal relaxation parameter

$\gamma_{2}$ : Concentration relaxation parameter

\section{Subscripts}

$f: \quad$ Fluid

p: Nanoparticle

$w$ : Condition at the surface

$\infty$ : Ambient condition

Superscripts

': Differentiation w. r. t. $\eta$.

\section{Data Availability}

Availability of data: The data available in this paper is available without any restriction.

\section{Conflicts of Interest}

The authors declare that they have no competing interests.

\section{Authors' Contributions}

All the authors have made substantive contributions to the article and assume full responsibility for its content. The authors read and approved the final manuscript.

\section{Acknowledgments}

The authors would like to express their deep Thanks are due to the referee for his/her careful reading and many valuable suggestions towards the improvement of the paper.

\section{References}

[1] I. Ameen, Z. Shah, S. Islam et al., "Hall and ion-slip effect on CNTS nanofluid over a porous extending surface through heat generation and absorption," Entropy, vol. 21, no. 8, pp. 801821, 2019.

[2] G. Rasool, T. Zhang, A. J. Chamkha, A. Shafiq, I. Tlili, and G. Shahzadi, "Entropy generation and consequences of binary chemical reaction on MHD Darcy-Forchheimer Williamson nanofluid flow over non-linearly stretching surface," Entropy, vol. 22, no. 1, p. 18, 2020.

[3] W. Ibrahim and T. Anbessa, "Mixed convection flow of a Maxwell nanofluid with hall and ion-slip impacts employing the spectral relaxation method," Heat Transfer, vol. 49, no. 5, pp. 3094-3118, 2020.

[4] I. Waini, A. Ishak, T. Groşan, and I. Pop, "Mixed convection of a hybrid nanofluid flow along a vertical surface embedded in a porous medium," International Communications in Heat and Mass Transfer, vol. 114, article 104565, 2020. 
[5] O. J. Fenuga, A. R. Hassan, and P. O. Olanrewaju, "Mixed convection in MHD flow and heat transfer rate near a stagnationpoint on a non-linear vertical stretching sheet," International Journal of Applied Mechanics and Engineering, vol. 25, no. 1, pp. 37-51, 2020.

[6] M. P. Mkhatshwa, S. S. Motsa, M. S. Ayano, and P. Sibanda, "MHD mixed convective nanofluid flow about a vertical slender cylinder using overlapping multi-domain spectral collocation approach," Case Studies in Thermal Engineering, vol. 18, article 100598, 2020.

[7] A. Jamaludin, K. Naganthran, R. Nazar, and I. Pop, "Thermal radiation and MHD effects in the mixed convection flow of $\mathrm{Fe}_{3} \mathrm{O}_{4}$-water ferrofluid towards a nonlinearly moving surface," Processes, vol. 8, no. 1, p. 95, 2020.

[8] Z. Shah, P. Kumam, and W. Deebani, "Radiative MHD Casson Nanofluid flow with activation energy and chemical reaction over past nonlinearly stretching surface through entropy generation," Scientific Reports, vol. 10, no. 1, pp. 4402-4414, 2020.

[9] A. Shafiq, G. Rasool, C. M. Khalique, and S. Aslam, "Second grade bioconvective Nanofluid flow with buoyancy effect and chemical reaction," Symmetry, vol. 12, no. 4, p. 621, 2020.

[10] A. Shafiq, G. Rasool, and C. M. Khalique, "Significance of thermal slip and convective boundary conditions in three dimensional rotating Darcy-Forchheimer Nanofluid flow," Symmetry, vol. 12, no. 5, p. 741, 2020.

[11] A. Shafiq, I. Zari, I. Khan, T. S. Khan, A. H. Seikh, and E. S. M. Sherif, "Marangoni driven boundary layer flow of carbon nanotubes toward a Riga plate," Frontiers in Physics, vol. 7, no. 215, pp. 1-11, 2020.

[12] A. Shafiq, I. Khan, G. Rasool, E. S. M. Sherif, and A. H. Sheikh, "Influence of single- and multi-wall carbon nanotubes on magnetohydrodynamic stagnation point nanofluid flow over variable thicker surface with concave and convex effects," Mathematics, vol. 8, no. 1, pp. 104-115, 2020.

[13] I. Khan, S. Fatima, M. Y. Malik, and T. Salahuddin, "Exponentially varying viscosity of magnetohydrodynamic mixed convection Eyring-Powell nanofluid flow over an inclined surface," Results in Physics, vol. 8, pp. 11941203, 2018.

[14] K. Ullah Sami, N. Ali, and Z. Abbas, "Soret and dufour effects on hydromagnetic flow of Eyring-Powell fluid over oscillatory stretching surface with heat generation/absorption and chemical reaction," Thermal Science, vol. 22, no. 1, Part B, pp. 533$543,2018$.

[15] M. Ishaq, G. Ali, S. I. A. Shah, Z. Shah, S. Muhammad, and S. A. Hussain, "Nanofluid film flow of Eyring-Powell fluid with magneto hydrodynamic effect on unsteady porous stretching sheet," Punjab University Journal of Mathematics, vol. 51, no. 2, pp. 131-153, 2019.

[16] M. Nawaz, I. H. Qureshi, and A. Shahzad, "Thermal performance of partially ionized Eyring-Powell liquid: a theoretical approach," Physica Scripta, vol. 94, no. 12, article 125209, 2019.

[17] B. Mallick and J. C. Misra, "Peristaltic flow of Eyring-Powell nanofluid under the action of an electromagnetic field," Engineering Science and Technology, vol. 22, no. 1, pp. 266-281, 2019.

[18] G. Rasool and T. Zhang, "Characteristics of chemical reaction and convective boundary conditions in Powell-Eyring nanofluid flow along a radiative Riga plate," Heliyon, vol. 5, no. 4, article e01479, 2019.
[19] M. Umar, R. Akhtar, Z. Sabir et al., "Numerical treatment for the three-dimensional Eyring-Powell fluid flow over a stretching sheet with velocity slip and activation energy," Advances in Mathematical Physics, vol. 2019, Article ID 9860471, 12 pages, 2019.

[20] B. Kumar and S. Srinivas, "Unsteady hydromagnetic flow of Eyring-Powell Nanofluid over an inclined permeable stretching sheet with joule heating and thermal radiation," Journal of Applied and Computational Mechanics, vol. 6, no. 2, pp. 259-270, 2020.

[21] T. Thumma and S. R. Mishra, "Effect of nonuniform heat source/sink, and viscous and joule dissipation on 3D Eyring-Powell nanofluid flow over a stretching sheet," Journal of Computational Design and Engineering, vol. 7, no. 4, pp. 412-426, 2020.

[22] Y. A. S. El-Masry, Y. A. Elmaboud, and M. A. Abdel-Sattar, "The impacts of varying magnetic field and free convection heat transfer on an Eyring-Powell fluid flow with peristalsis: VIM solution," Journal of Taibah University for Science, vol. 14, no. 1, pp. 19-30, 2020.

[23] Z. Khan, H. U. Rasheed, T. Abbas et al., “Analysis of EyringPowell fluid flow used as a coating material for wire with variable viscosity effect along with thermal radiation and joule heating," Crystals, vol. 10, no. 3, p. 168, 2020.

[24] M. V. Krishna and A. J. Chamkha, "Hall and ion slip effects on Unsteady MHD Convective Rotating flow of Nanofluids-Application in Biomedical Engineering," Journal of the Egyptian Mathematical Society, vol. 28, no. 1, pp. 1-15, 2020.

[25] C. H. Baby Rani, N. Vedavathi, K. S. Balamurugan, and G. Dharmaiah, "Hall and ion slip effects on AG - WATER based MHD nanofluid flow over a semi-infinite vertical plate embedded in a porous medium," Frontiers in Heat and Mass Transfer, vol. 14, no. 6, pp. 1-11, 2020.

[26] M. Nawaz, S. Rana, I. H. Qureshi, and T. Hayat, "Threedimensional heat transfer in the mixture of nanoparticles and micropolar MHD plasma with Hall and ion slip effects," AIP Advances, vol. 8, no. 10, article 105109, 2018.

[27] M. Veera Krishna and A. J. Chamkha, "Hall and ion slip effects on MHD rotating boundary layer flow of nanofluid past an infinite vertical plate embedded in a porous medium," Results in Physics, vol. 15, article 102652, 2019.

[28] M. Bilal and M. Ramzan, "Hall current effect on unsteady rotational flow of carbon nanotubes with dust particles and nonlinear thermal radiation in Darcy-Forchheimer porous media," Journal of Thermal Analysis and Calorimetry, vol. 138, no. 5, pp. 3127-3137, 2019.

[29] M. Rafiq, H. Yasmin, T. Hayat, and F. Alsaadi, "Effect of hall and ion-slip on the peristaltic transport of nanofluid: a biomedical application," Chinese Journal of Physics, vol. 60, pp. 208-227, 2019.

[30] N. Feroz, Z. Shah, S. Islam, E. Alzahrani, and W. Khan, "Entropy generation of carbon nanotubes flow in a rotating channel with hall and ion-slip effect using effective thermal conductivity model," Entropy, vol. 21, no. 1, p. 52, 2019.

[31] I. H. Qureshi, M. Nawaz, and A. Shahzad, "Numerical study of dispersion of nanoparticles in magnetohydrodynamic liquid with Hall and ion slip currents," AIP Advances, vol. 9, no. 2, article 025219, 2019.

[32] J. B. J. Fourier, Théorie analytique de la chaleur, F. Didot, 1822.

[33] C. Cattaneo, "Sulla conduzione del calore," Atti Del Semin Matem E Fis Della Univ Modena, vol. 3, pp. 83-101, 1948. 
[34] C. I. Christov, "On frame indifferent formulation of the Maxwell-Cattaneo model of finite-speed heat conduction," Mechanics Research Communications, vol. 36, no. 4, pp. 481486, 2009.

[35] A. Shafiq, S. Jabeen, T. Hayat, and A. Alsaedi, "CattaneoChristov heat flux model for squeezed flow of third grade fluid," Surface Review and Letters, vol. 24, no. 7, article 1750098, p. 11, 2017.

[36] A. Naseem, A. Shafiq, L. Zhao, and M. U. Farooq, "Analytical investigation of third grade nanofluidic flow over a Riga plate using Cattaneo-Christov model," Results in Physics, vol. 9, pp. 961-969, 2018.

[37] A. Rauf, Z. Abbas, S. A. Shehzad, A. Alsaedi, and T. Hayat, "Numerical simulation of chemically reactive Powell-Eyring liquid flow with double diffusive Cattaneo-Christov heat and mass flux theories," Applied Mathematics and Mechanics, vol. 39, no. 4, pp. 467-476, 2018.

[38] R. V. M. S. S. Kiran Kumar and S. V. K. Varma, "MHD Boundary Layer Flow of Nanofluid Through a Porous Medium Over a Stretching Sheet with Variable Wall Thickness: Using Cattaneo-Christov Heat Flux Model," Journal of Theoretical and Applied Mechanics, vol. 48, no. 2, pp. 72-92, 2018.

[39] T. Hayat and S. Nadeem, "Flow of 3D Eyring-Powell fluid by utilizing Cattaneo-Christov heat flux model and chemical processes over an exponentially stretching surface," Results in Physics, vol. 8, pp. 397-403, 2018.

[40] M. Ramzan, A. Liaquet, S. Kadry, S. Yu, Y. Nam, and D. Lu, "Impact of second-order slip and double stratification coatings on 3D MHD Williamson nanofluid flow with Cattaneo-Christov heat flux," Coatings, vol. 9, no. 12, p. 849, 2019.

[41] G. Rasool and T. Zhang, "Darcy-Forchheimer nanofluidic flow manifested with Cattaneo-Christov theory of heat and mass flux over non-linearly stretching surface," PLoS One, vol. 14, no. 8, article e0221302, 2019.

[42] N. Muhammad, S. Nadeem, and A. Issakhov, "Finite volume method for mixed convection flow of Ag-ethylene glycol nanofluid flow in a cavity having thin central heater," Physica A: Statistical Mechanics and its Applications, vol. 537, article 122738, 2020.

[43] Z. Shah, A. Tassaddiq, S. Islam, A. M. Alklaibi, and I. Khan, "Cattaneo-Christov heat flux model for three-dimensional rotating flow of SWCNT and MWCNT Nanofluid with Darcy-Forchheimer porous medium induced by a linearly stretchable surface," Symmetry, vol. 11, no. 3, p. 331, 2019.

[44] S. R. R. Reddy, P. Bala Anki Reddy, and A. M. Rashad, "Activation energy impact on chemically reacting Eyring-Powell Nanofluid flow over a stretching cylinder," Arabian Journal for Science and Engineering, vol. 45, no. 7, pp. 5227-5242, 2020.

[45] T. Muhammad, H. Waqas, S. A. Khan, R. Ellahi, and S. M. Sait, "Significance of nonlinear thermal radiation in 3D EyringPowell nanofluid flow with Arrhenius activation energy," Journal of Thermal Analysis and Calorimetry, pp. 1-16, 2020.

[46] W. Ibrahim and T. Anbessa, "Three-Dimensional MHD Mixed Convection Flow of Casson Nanofluid with Hall and Ion Slip Effects," Mathematical Problems in Engineering, vol. 2020, Article ID 8656147, 15 pages, 2020.

[47] S. S. Motsa, P. Sibanda, J. M. Ngnotchouye, and G. T. Marewo, "A spectral relaxation approach for unsteady boundary-layer flow and heat transfer of a nanofluid over a permeable stretching/shrinking sheet," Advances in Mathematical Physics, vol. 2014, Article ID 564942, 10 pages, 2014.
[48] I. S. Oyelakin, S. Mondal, and P. Sibanda, "Unsteady Casson nanofluid flow over a stretching sheet with thermal radiation, convective and slip boundary conditions," Alexandria Engineering Journal, vol. 55, no. 2, pp. 1025-1035, 2016.

[49] S. Abdul Gaffar, V. Ramachandra Prasad, and E. Keshava Reddy, "MHD free convection flow of Eyring-Powell fluid from vertical surface in porous media with Hall/ionslip currents and ohmic dissipation," Alexandria Engineering Journal, vol. 55, no. 2, pp. 875-905, 2016.

[50] L. N. Trefethen, "Spectral Methods in MATLAB," in vol. 10 of Software, Environments, and Tools, SIAM, Philadelphia, PA, USA, 2000.

[51] A. Noghrehabadi, R. Pourrajab, and M. Ghalambaz, "Effect of partial slip boundary condition on the flow and heat transfer of nanofluids past stretching sheet prescribed constant wall temperature," International Journal of Thermal Sciences, vol. 54, pp. 253-261, 2012.

[52] M. Goyal and R. Bhargava, "Finite element solution of doublediffusive boundary layer flow of viscoelastic nanofluids over a stretching sheet," Computational Mathematics and Mathematical Physics, vol. 54, no. 5, pp. 848-863, 2014.

[53] W. Ibrahim and G. Gadisa, "Finite element method solution of boundary layer flow of Powell-Eyring nanofluid over a nonlinear stretching surface," Journal of Applied Mathematics, vol. 2019, Article ID 3472518, 16 pages, 2019. 